\title{
MR-labelled liposomes and focused ultrasound for spatiotemporally controlled drug release in triple negative breast cancers in mice
}

\author{
Maral Amrahli ${ }^{1}$, Miguel Centelles ${ }^{1}$, Paul Cressey ${ }^{1}$, Martynas Prusevicius ${ }^{1}$, Wladyslaw Gedroyc ${ }^{2}$, Xiao Yun Xu ${ }^{3}$, \\ Po-Wah So ${ }^{4}$, Michael Wright ${ }^{1 \#}$, and Maya Thanou ${ }^{1 \# \bowtie}$ \\ 1. School of Cancer \& Pharmaceutical Sciences, King's College London, U.K. \\ 2. Department of Academic Surgery, Imperial College London, U.K. \\ 3. Department of Chemical Engineering, Imperial College London, U.K. \\ 4. Department of Neuroimaging, King's College London, U.K. \\ \#Contributed equally to this work. \\ $\triangle$ Corresponding author: Institute of Pharmaceutical Science, Franklin Wilkins Building, 150 Stamford Street, London, SE1 9NH, U.K.; E-mail: maya.thanou@kcl.ac.uk; \\ mjl.wright@gmail.com.
}

(C) The author(s). This is an open access article distributed under the terms of the Creative Commons Attribution License (https://creativecommons.org/licenses/by/4.0/). See http://ivyspring.com/terms for full terms and conditions.

Received: 2020.08.21; Accepted: 2020.11.30; Published: 2021.01.01

\begin{abstract}
Rationale: Image-guided, triggerable, drug delivery systems allow for precisely placed and highly localised anti-cancer treatment. They contain labels for spatial mapping and tissue uptake tracking, providing key location and timing information for the application of an external stimulus to trigger drug release. High Intensity Focused Ultrasound (HIFU or FUS) is a non-invasive approach for treating small tissue volumes and is particularly effective at inducing drug release from thermosensitive nanocarriers. Here, we present a novel MR-imageable thermosensitive liposome (iTSL) for drug delivery to triple-negative breast cancers (TNBC).

Methods: A macrocyclic gadolinium-based Magnetic Resonance Imaging (MRI) contrast agent was covalently linked to a lipid. This was incorporated at $30 \mathrm{~mol} \%$ into the lipid bilayer of a thermosensitive liposome that was also encapsulating doxorubicin. The resulting iTSL-DOX formulation was assessed for physical and chemical properties, storage stability, leakage of gadolinium or doxorubicin, and thermal- or FUS-induced drug release. Its effect on MRI relaxation time was tested in phantoms. Mice with tumours were used for studies to assess both tumour distribution and contrast enhancement over time. A lipid-conjugated near-infrared fluorescence (NIRF) probe was also included in the liposome to facilitate the real time monitoring of iTSL distribution and drug release in tumours by NIRF bioimaging. TNBC (MDA-MB-231) tumour-bearing mice were then used to demonstrate the efficacy at retarding tumour growth and increasing survival.

Results: iTSL-DOX provided rapid FUS-induced drug release that was dependent on the acoustic power applied. It was otherwise found to be stable, with minimum leakage of drug and gadolinium into buffers or under challenging conditions. In contrast to the usually suggested longer FUS treatment we identified that brief $(\sim 3$ min) FUS significantly enhanced iTSL-DOX uptake to a targeted tumour and triggered near-total release of encapsulated doxorubicin, causing significant growth inhibition in the TNBC mouse model. A distinct reduction in the tumours' average $T_{1}$ relaxation times was attributed to the iTSL accumulation.

Conclusions: We demonstrate that tracking iTSL in tumours using MRI assists the application of FUS for precise drug release and therapy.
\end{abstract}

Key words: liposome; MRI; focused ultrasound; doxorubicin; triple-negative breast cancer

\section{Introduction}

More than 40 years ago it was suggested that the effectiveness of anticancer drugs might be enhanced by the application of localised mild hyperthermia [1]. Recent technological advances allow for the application of heat to deep seated tissues, with high spatial precision and accurate thermal control. High Intensity Focused Ultrasound (HIFU or FUS) is the most accurate non-invasive hyperthermia method [2]. 
Clinical FUS was developed to deposit ultrasound energy within small tissue volumes [3]. Its extension to Magnetic Resonance guided (MRg) FUS allows for highly accurate spatial localisation and is clinically used for precise ablative treatment of solid tumours and neurological disorders [4]. A less clinically investigated application is the use of MR-guided FUS hyperthermia for targeted drug delivery using thermoresponsive liposomes $[5,6]$.

Liposomes are in widespread clinical use as nanocarriers that offer a high level of multifunctionality and versatility [7]. Thermosensitive liposomes (TSL) have been developed as drug delivery systems able to release their cargo almost instantly when warmed to $\sim 42^{\circ} \mathrm{C}[8,9]$. TSL have been tested in small and large animals and have shown great efficiency when combined with hyperthermia $[10,11]$. A potentially valuable (but not yet fully developed) modification would be the inclusion of MRI contrast agents (CA) to allow for real-time image guidance, uptake tracking, and monitoring of the drug release within a lesion [12-14]. Such MR-labelled carriers also offer anatomical guidance, feedback, and spatiotemporal control of the applied FUS - offering the opportunity of real-time treatment for large tumours and potentially their metastases [15].

It has been shown that FUS can be combined with TSL loaded with gadolinium-based paramagnetic MRI contrast agents [16]. A study by Kono et al. has reported on gadolinium DOTA $(1,4,7,10$-tetraazacyclododecane-1,4,7,10-tetraacetic acid) chelates introduced in a dendron structure attached to the lipid membrane of liposomes coated with thermossensitive polymer [12]. But usually gadoliniummacrocyclic CA (FDA approved and common in clinical imaging) are encapsulated into the aqueous TSL core [17]. The TSL release these small molecules after heating, giving an MR-visible confirmation of any concurrent drug release - a technique dubbed 'dose painting' [18]. The effect is transient since the freed CA rapidly diffuses away from the tumour. Lipid membrane-bound CA have the advantage here as they can be monitored throughout a treatment and for longer timeframes [12].

ThermoDox ${ }^{\circledR}$ is a doxorubicin-bearing TSL and was the first 'responsive' nanocarrier that moved to clinical trials [9]. A recently completed Phase-III (NCT00617981) evaluated its efficacy in combination with radiofrequency ablation in patients with hepatocellular carcinoma [19], while TARDOX is a published Phase-I that demonstrated the safety of using FUS to trigger drug release [20]. This showed that $60 \mathrm{~min}$ of FUS treatment significantly increased doxorubicin concentration in tumours. We previously presented dual-labelled (near-infrared fluorescence and MRI) thermosensitive liposomes for imaging (iTSL) as a tool that provides insight on the mechanism of drug release and the temporal and spatial distribution of drug nanocarriers in tumours $[21,22]$. Near-infrared fluorescence (NIRF) imaging tracked the liposomes in real time, allowing assessment of their distribution and this information was used to decide the time and duration of FUS treatments. We demonstrated that FUS caused an increase in iTSL uptake to tumours and triggered coordinated drug release (using topotecan). Surprisingly, only brief FUS treatments were needed to significantly modify liposome distribution and induce rapid drug release [21,22].

Here, we investigate the ability of iTSL loaded with doxorubicin (iTSL-DOX) to be tracked by MRI in mice and assess if brief FUS treatments can significantly improve the drug's anti-tumour efficacy. We modify the composition of a lysolipid-containing TSL with a gadolinium-DOTA lipid to allow image-based tracking in tumours post-injection. We synthesised and incorporated the MR-labelled lipid (Gd.DOTA.DSA) in a liposome bilayer at almost 30 $\mathrm{mol} \%$ of the lipid composition and investigated the effects of this on thermo-sensitivity and the ability to encapsulate/release doxorubicin. After optimisation of the composition, we assessed iTSL-DOX for drug and $\mathrm{Gd}^{3+}$ leakage and for stability and thermal release performance after long term storage (3 months). Using iTSL-DOX in an acoustic gel block embedded capillary tube model, we demonstrate that drug release can be instantaneous and is strongly dependent on the applied FUS power. To allow for the tracking of iTSL-DOX in vivo a small amount of lipid-conjugated NIRF probe (CF750-DSDA; 0.05 mol\%) was also included. This label provided valuable information during development, although NIRF is unlikely to be used in the clinic due to limited tissue penetration. NIRF-imaging in live mice showed iTSL-DOX accumulation and doxorubicin release in tumours during the application of FUS. Efficacy on tumours was demonstrated on a triple-negative breast cancer (TNBC; MDA-MB-231) murine model. The potential advantages of MRI tracking are shown by assessing liposome effects on phantom and tumour $\mathrm{T}_{1}$ relaxivity (a measure of contrast enhancement).

\section{Material and Methods}

\section{Lipid synthesis}

Gadolinium (III) 2-(4,7-bis-carboxymethyl-10$[(N, \quad N$-distearylamidomethyl- $N$ '-amidomethyl $]-1,4,7$, 10-tetraazacyclododec-1-yl) acetic acid (Gd.DOTA. DSA; MR-labelled lipid) and N-CF750-N,N-distearylamidomethylamine (CF750.DSA; NIRF-labelled lipid) 
were synthesised, purified, and confirmed according to our previous report [21] (Scheme S1; CF750 succinimidyl ester (Biotium, CA, USA) was previously called XL750-NHS).

\section{Preparation of iTSL-DOX}

1,2-Dipalmitoyl-sn-glycero-3-phosphocholine

(DPPC; 16:0 PC), 1,2-distearoyl-sn-glycero-3phosphocholine (DSPC; 18:0 PC), 1-stearoyl-snglycero-3-phosphocholine (MSPC; 18:0 lyso-PC) and ( $\omega$-methoxy-polyethyleneglycol $\left.{ }^{2000}\right)-N$-carboxy-1,2-dis tearoyl-sn-glycero-3-phosphoethanolamine (DSPEPEG $^{2000}$ ) were purchased from Avanti Polar Lipids (AL, USA) or Sigma Aldrich (MO, USA). Lipids were stored at $-20{ }^{\circ} \mathrm{C}$ as aliquots of $10-20 \mathrm{mg} / \mathrm{mL}$ in chloroform, methanol or methanol:chloroform 1:1 $(\mathrm{v} / \mathrm{v})$ according to their solubility. Appropriate amounts of each were combined to give a mixture of Gd.DOTA.DSA/DPPC/DSPC/MSPC/DSPE-PEG 2000 /CF750.DSA at 30/54/5/5/6/0.05 $\mathrm{mol} \%$ and $30 \mathrm{mg}$ total lipid per batch. The solvent was removed using a rotary evaporator and the resulting film dried overnight in vacuo. Further preparation stages were carried out in a sterilised fume hood with all equipment and materials being single use, sterilised by autoclave, or wiped down with ethanol. Buffers were sterilised by filtration $(0.2 \mu \mathrm{m})$.

Each lipid film was hydrated with $1 \mathrm{~mL}$ of loading buffer (300 mM ammonium phosphate aq.; $\mathrm{pH}$ 4.0) and fragmented using repeated ( 10×) freeze/thaw from liquid nitrogen to a water sonication bath at $53^{\circ} \mathrm{C}$, followed by further sonication, and extrusion $3 \times$ through a $100 \mathrm{~nm}$ pore polycarbonate membrane using a gas powered extruder (LIPEX, Northern Lipids, Canada) heated to $52{ }^{\circ} \mathrm{C}$. The iTSL was then exchanged to storage buffer (50 mM HEPES aq. with $5 \mathrm{w} \%$ glucose; $\mathrm{pH} 7.4$ ) using a PD10 size-exclusion column (Amersham, UK). They were loaded with doxorubicin using a thermocycler to provide accurate temperatures. Concentrated doxorubicin $\mathrm{HCl}$ aq. (stored frozen) was added to 1.2 $\mathrm{mg} / \mathrm{mL}$, then incubated at $38.7{ }^{\circ} \mathrm{C}$ for $1 \mathrm{~h} 50 \mathrm{~m}$. Sterility was checked by inoculating LB-agar plates with 100-fold diluted iTSL-DOX and incubation overnight at $37^{\circ} \mathrm{C}$. No bacterial colonies were seen, unlike plates inoculated with non-sterile storage buffer.

\section{Dynamic Light Scattering (DLS)}

iTSL-DOX batches were routinely assessed using a Nanoseries Nano ZS (Malvern Panalytical, UK). Samples were diluted 1:20 (v/v) using storage buffer at $25^{\circ} \mathrm{C}$ and size modelling used default solute and particle parameters.

\section{Doxorubicin quantification by HPLC}

iTSL-DOX absorbance and fluorescence profiles were collected using an Infinite 200 Pro plate reader (Tecan, Switzerland). Expected absorbance peaks were seen at $480 \mathrm{~nm}$ (doxorubicin), $750 \mathrm{~nm}$ (CF750 NIRF dye) and fluorescence peaks at $\mathrm{Ex}_{480} / \mathrm{Em}_{590}$ (doxorubicin), $\mathrm{Ex}_{750} / \mathrm{Em}_{800}$ (CF750). Dilute iTSL-DOX shows a 3-5x increase in doxorubicin fluorescence intensity on incubation at temperatures above $40^{\circ} \mathrm{C}$, due to release of encapsulated drug and resulting de-quenching. The NIR fluorescence of the iTSL is not affected by this.

Total doxorubicin concentration was assessed by reverse-phase HPLC using an Agilent 1100 equipped with a multi-wavelength diode array detector, a 1260 Infinity fluorescence detector (all from Agilent, CA, USA), and a $5 \mathrm{~cm}$ Hypersil C18 $5 \mu \mathrm{m}$ reverse-phase column (Thermo Fisher, MA, USA). Solvents were: water with $0.1 \mathrm{v} \%$ trifluoroacetic acid (A) and acetonitrile (B). Gradient was: $0 \mathrm{~min} 0 \% \mathrm{~B}, 1.5 \mathrm{~min} 0 \%$, $5 \operatorname{min~} 50 \%, 6 \min 50 \%, 7 \mathrm{~min} 0 \%, 8.5 \mathrm{~min} 0 \%$ and a flow rate of $3.5 \mathrm{~mL} / \mathrm{min}$. Doxorubicin gave a single fluorescence peak $(4.1 \mathrm{~min})$ the area being used to estimate concentration after calibration (0-200 ng range; $\mathrm{R}$ 0.999) against a doxorubicin- $\mathrm{HCl}$ European Pharmacopoeia Reference Standard.

During loading optimisation, the lipid:drug ratio was assessed by gel-filtration HPLC using a Tricorn 5/100 column (GE Life Sciences, PA, USA) packed with Sephadex G20 slurry and run in water at 1 $\mathrm{mL} / \mathrm{min}$. Total lipid concentration determination used a modified Stewart assay: iTSL-DOX samples (50 $\mu \mathrm{L})$ were mixed with water $(150 \mu \mathrm{L})$ and $\mathrm{MeOH}: \mathrm{CHCl}_{3}, 1: 1(\mathrm{v} / \mathrm{v} ; 200 \mu \mathrm{L})$ then vortex mixed giving an emulsion. This was centrifuged (4000 g; 2 min) to fully separate the organic and aqueous layers. An aliquot $(70 \mu \mathrm{L})$ of the organic layer was combined with Stewart reagent (5 $\mu \mathrm{L} ; \mathrm{FeCl}_{3} / \mathrm{NH}_{4} \mathrm{SCN}$ aq.), emulsified and centrifuged again. Finally, an organic aliquot $(50 \mu \mathrm{L})$ was transferred to a glass 96-well plate and the $455 \mathrm{~nm}$ absorbance was measured. Calibration was against matched lipid suspensions prepared with sonication alone to avoid extrusion loses.

\section{Gadolinium quantification by total-reflection X-ray fluorescence (TXRF)}

This was performed on a PICOFOX ${ }^{\mathrm{TM}_{-} \mathrm{S} 2}$ (Bruker, MA, USA) [23] with samples mixed 1:1 (v/v) with diluted gallium aq. (TraceCERT ${ }^{\circledR}$ certified reference) as an internal standard and using Ultrapure water and trace metal analysis grade reagents. Tissues were digested with nitric acid $(150 \mu \mathrm{L} ; 68 \mathrm{w} \%$ aq.) and hydrogen peroxide $(50 \mu \mathrm{L} ; 30 \mathrm{w} \%$ aq.) in tightly sealed plastic tubes at $70{ }^{\circ} \mathrm{C}$ overnight, while 
heparin-treated blood and liposome samples were analysed directly. Sample/standard mixtures were homogenised, 5-10 $\mu \mathrm{L}$ applied onto siliconised quartz disc sample carriers (Bruker Nano GMbH, Germany) and oven dried at $70{ }^{\circ} \mathrm{C}$. TXRF results were collected in triplicate over $1000 \mathrm{~s}$ using excitation settings of 50 $\mathrm{kV}$ and $600 \mathrm{~mA}$. Spectra were inspected and all significant peaks identified, prior to deconvolution using PICOFOX ${ }^{\mathrm{TM}}$ V 7.5.3.0 (Bruker Nano GmbH).

\section{iTSL-DOX thermosensitivity}

Assessment of lipid membrane thermosensitivity used liquid phase differential scanning calorimetry (DSC, TA Instruments, DE, USA) to record at least two rounds of heating/cooling cycles $\left(25-70{ }^{\circ} \mathrm{C}\right.$ at 1 ${ }^{\circ} \mathrm{C} / \mathrm{min}$; $3 \mathrm{~atm}$ ) from samples of each iTSL-DOX batch against degassed plain buffer.

Thermally-induced doxorubicin release was assessed by the increase in $\mathrm{Ex}_{480} / \mathrm{Em}_{590}$ fluorescence seen as it escapes from the highly self-quenched encapsulated environment after incubation $>42{ }^{\circ} \mathrm{C}$. Studies used separate samples (100 $\mu \mathrm{L}$; triplicate) diluted 1:100 (v/v) in storage buffer, or $50 \mathrm{v} \%$ fetal bovine serum (FBS) as a blood analogue. Fluorescence intensity readings were normalised against iTSLDOX, unheated and also after $5 \mathrm{~min}$ at $50{ }^{\circ} \mathrm{C}$ to assess release $\%$.

\section{iTSL-DOX gadolinium retention}

The potential for 'leakage' of $\mathrm{Gd}^{3+}$ from chelation to Gd.DOTA.DSA was investigated using a dialysis assay. This measured the escape of $\mathrm{Gd}^{3+}$ from an inner chamber of a Slide-A-Lyzer (MINI Dialysis Device, 10K MWCO, Thermo Scientific) containing either iTSL or $0.2 \mathrm{mg} / \mathrm{mL}$ aq. gadolinium standard (TraceCERT®) to an outer cuvette containing either Ultrapure water or $50 \mathrm{v} \%$ FBS water (at $4{ }^{\circ} \mathrm{C}$ to avoid protein aggregation). The cuvettes were equipped with small magnetic beads, placed on a magnetic stirrer then $10 \mu \mathrm{L}$ samples taken over $48 \mathrm{~h}$ and analysed by TXRF as above. Doxorubicin was omitted as it blocked the dialysis membrane.

\section{FUS-induced doxorubicin release}

A gel block embedded capillary model was developed that allowed for fluorescence visualisation of doxorubicin release from iTSL-DOX while under FUS using a preclinical small animal FUS system (Therapy and Imaging Probe System, 'TIPS'; Philips Research, Netherlands; Figure S1 -2).

Optically transparent polyacrylamide gel with suitable acoustic properties was cast into cylindrical blocks. A fine plastic flow-tube $(0.8 \mathrm{~mm}$ inner diameter) was placed within the gel block positioned to pass through the focal volume of the transducer. A fine-wire thermocouple (T150A; Linton
Instrumentation, U.K.) was threaded through the flow-tube such that the sensing tip was placed next to the focus. The other end of the flow-tube was connected to a syringe of iTSL-DOX (200-fold dilution into storage buffer) pumped by a driver at up to 100 $\mu \mathrm{L} / \mathrm{min}$. A pad of acoustic foam was placed below the gel block to absorb the post-focus FUS and prevent the formation of standing waves. The gel block was then immersed in a water bath at $\sim 35{ }^{\circ} \mathrm{C}$ and illuminated from the sides, either by white light or $3 \times$ blue LED (460 nm peak, $10 \mathrm{~W}$; LED Engin LZ4-20B200; Osram, Germany). Imaging was down the barrel of the FUS transducer and used a Blackfly video camera (BFLY-PGE-13E4M-CS; FLIR Systems, OR, USA) equipped with a $550 \mathrm{~nm}$ long-pass dichroic glass filter. A pulsed FUS program was used, setting the transducer to $1.4 \mathrm{MHz}$, acoustic power up to $18 \mathrm{~W}$, duty cycle $25 \%$, PRF $0.5 \mathrm{~Hz}$ (equivalent to $0.5 \mathrm{~s}$ on, 1.5 $\mathrm{s}$ off). Processing of the resulting videos concentrated on a small rectangular region-of-interest (ROI) placed just downstream of the FUS focus. After conversion of the video to individual frames, the average ROI pixel intensity was calculated using ImageJ v1.4 (National Institutes of Health, USA) and plotted against frame time. The measured temperatures were then collated and aligned.

\section{MRI relaxivity}

The ability for iTSL to enhance $T_{1}$ relaxation was investigated on two horizontal bore preclinical scanners; a VNMRS (Agilent, Walnut Creek, California, USA) at $7 \mathrm{~T}$, and a BioSpec (Bruker Biospin, Ettlingen, Germany) at 9.4 T. Both used a quadrature volume radio-frequency coil of inner diameter of $39 \mathrm{~mm}$ (RAPID Biomedical $\mathrm{GmbH}$, Rimpar, Germany).

Both MRI studies were performed on liquid phantoms with various concentrations of iTSL (with/without DOX) and diluted Gadovist ${ }^{\circledR}$ (a clinically-relevant control). iTSL-DOX and 'released' iTSL-DOX (previously incubated at $45^{\circ} \mathrm{C}$ for $3 \mathrm{~min}$ ) phantoms were used for the $7 \mathrm{~T}$ study. $\mathrm{T}_{1}$-weighted spin-echo sequence was performed at room temperature with the following parameters: TR 12.06, $20,30,40,50,60,80,100,120,150,180,200,250,300$, $350,400,450,500,700,850,1000,1200,1500,1700$, 2000, 5000, and $10000 \mathrm{~ms}$; TE $8 \mathrm{~ms}$; average of 2; matrix size $128 \times 128$; and FOV $40 \times 40 \mathrm{~mm}$. $\mathrm{T}_{1}$ maps were calculated from the relaxometry data by pixel-by-pixel fitting to Equation 1 using ImageJ.

$$
M_{i}=M_{0}\left(1-e^{\left(-T R / T_{1}\right)}\right)
$$

Equation 1: Standard saturation recovery equation used to determine the $T_{1}$ longitudinal 
relaxation time; where $M_{i}$ is signal intensity, TR repetition time, and $\mathrm{M}_{0}$ proton density.

$\mathrm{T}_{1}$-relaxometry of iTSL-NO-DOX phantoms at a magnetic field of $9.4 \mathrm{~T}$ was carried out in a similar fashion. A fast spin-echo sequence was performed using: TR 45, 50, 60, 80, 100, 120, 150, 180, 200, 350, $450,500,750,850,900,1000,1200,1500,1700,2000$, 3000, 5000 and $10000 \mathrm{~ms}$; TE $11 \mathrm{~ms}$; and 2 averages. Sufficient numbers of $2 \mathrm{~mm}$ thick coronal imaging slices were used to image volumes of interest with field of view (FOV) $26 \times 26 \mathrm{~mm}$ and matrix size $128 \times 128 . T_{1}$ maps were generated from raw data by pixel-by-pixel non-linear fitting to Equation 1 using JIM v8.0 (Xinapse Systems, Alwincle, UK).

In both studies the analysis of images included a selection of ROI drawn by hand and placed on the maps to obtain mean $T_{1}$ relaxation times. The longitudinal relaxivities $\left(\mathrm{r}_{1}\right)$ were calculated from a linear relationship of the relaxation rate as a function of the gadolinium concentrations (Equation 2) using Prism v8.2 (GraphPad Software, San Diego, SA, USA). Gadolinium concentrations from the iTSL used in the phantoms were derived by TXRF quantification as described above.

$$
r_{1}=\frac{\left(R_{1}-R_{1(0)}\right)}{[C A]}
$$

Equation 2: Calculation of molar relaxivity $\left(\mathrm{r}_{1}\right)$; where $R_{1}$ is relaxation rate (equivalent to $1 / T_{1}$ ), $R_{1(0)}$ is the relaxation rate of the buffer, and [CA] is the contrast agent concentration (in this case $\mathrm{Gd}^{3+}$ ) [24].

\section{Animal studies}

All animal procedures were conducted under the U.K. Home Office regulations and the Guidance for the Operation of Animals (Scientific Procedures) Act (1986). Female 4-6 week old athymic nude mice and CD-1 mice were from Envigo (Huntingdon, UK) and female 4-6 week old Severe Combined Immunodeficiency Hairless Outbred (SHO) mice were from Charles River Laboratories (Wilmington, MA, USA). Unless otherwise stated, injections were given via an i.v.-cannula implanted in the tail vein, and used a syringe driver $(100 \mu \mathrm{L} / \mathrm{min})$.

\section{Gadolinium and doxorubicin pharmacokinetics}

Gadolinium clearance study used iTSL $(200 \mu \mathrm{L}$; [Gd] $1.3 \mathrm{mg} / \mathrm{mL})$ administered to CD-1 mice $(\mathrm{n}=6)$ with blood samples ( $25 \mu \mathrm{L}$ each; over $4 \mathrm{~h}$ ) collected from the caudal vein and transferred into pre-weighed vials along with heparin $(2 \mu \mathrm{L})$. These were initially kept on ice and then frozen for storage. Portions $(10 \mu \mathrm{L})$ of each were mixed with a gallium internal standard $(10 \mu \mathrm{L} ; 4 \mathrm{mg} / \mathrm{L})$ and polyvinyl alcohol aq. $(10 \mu \mathrm{L} ; 1.2 \mathrm{~g} / \mathrm{L})$ before analysis by TXRF as previously described.
In a separate study, CD-1 mice $(\mathrm{n}=7)$ were given an i.v. dose of iTSL-DOX (4 mg/ $\mathrm{kg}$ doxorubicin) and blood samples ( $55 \mu \mathrm{L}$ each; over $3 \mathrm{~h}$ ) collected. These were handled as above but centrifuged $(4,500 \mathrm{rpm} ; 5$ min; $4{ }^{\circ} \mathrm{C}$ ) to isolate plasma before freezing. Doxorubicin extraction was carried out by addition of acetonitrile, centrifugation $\left(10,000 \mathrm{rpm} ; 5 \mathrm{~min} ; 4^{\circ} \mathrm{C}\right)$ and analysis of the supernatant by LC-MS/MS, using daunorubicin- $\mathrm{HCl}$ as an internal standard. This used a Hypersil Gold aQ $50 \times 2.1 \mathrm{~mm} 3 \mu$ reverse phase column (Thermo Scientific; Waltham, MA, U.S.A.) and TSQ Quantum Access mass spectrometer (+ve ESI) with indicative selected reaction monitoring transitions $544.170 \rightarrow 378.940,544.170 \rightarrow 396.950$ for doxorubicin or $528.18 \rightarrow 328.9$ for daunorubicin and with calibration against doxorubicin- $\mathrm{HCl}(20-50,000$ $\mathrm{ng} / \mathrm{mL}$ ).

\section{Tumour induction}

MDA-MB-231 cells $\left(6 \times 10^{6}\right.$ per tumour) were suspended in PBS and then mixed 1:1 (v/v) with Geltrex Matrix (ThermoFisher). The mixture was placed subcutaneously (s.c.) on the dorsal haunch of each mouse, with either single tumour (right side only) or double tumour (both sides) models prepared according to the study needs. Tumours were considered ready for FUS treatment once they had reached 5-6 $\mathrm{mm} \varnothing$ when measured with a digital calliper.

\section{Murine FUS}

Ultrasound was applied using a Therapy and Imaging Probe System preclinical FUS (TIPS; Philips Research, Netherlands). Mice were anaesthetised with isoflurane/oxygen and placed on a warmed gel pad over an acoustic foam mat to absorb unwanted ultrasound. Two fine-wire thermocouples (T150A; Linton Instrumentation, U.K.) were carefully implanted around the target tumour. Warmed and degassed (by centrifugation) ultrasound gel was used to fill all air gaps between the pad, the mouse, and the TIPS window. Normally the TIPS window was placed $8.0 \mathrm{~mm}$ above the tumour surface and FUS was applied at $1.3 \mathrm{MHz}$, using a $100 \%$ duty cycle. This placed the focus above the skin surface, minimising the risk of skin burns or unwanted tumour ablation but allowing for efficient FUS-induced hyperthermia. Tumour temperatures were measured at $50 \mathrm{~ms}$ resolution and TIPS acoustic power settings were adjusted manually $(10-20 \mathrm{~W})$ to converge on the target $\left(42 / 43^{\circ} \mathrm{C}\right)$ and this could then be maintained with little variation. A normal protocol consisted of two rounds of FUS: $20 \mathrm{~min}$ pre-injection and $45 \mathrm{~min}$ post-injection. 


\section{iTSL-DOX biodistribution}

Near-infrared fluorescence (NIRF) and gadolinium quantification was used for imaging the iTSL and to analyse tumour content. Mice bearing single tumours were anesthetised and iTSL $(200 \mu \mathrm{L}$; [Gd]: $0.56 \mathrm{mg} / \mathrm{mL}$ ) administered i.v. via the tail vein by slow infusion ( $35 \mu \mathrm{L} / \mathrm{min})$. The FUS group received two rounds of FUS (20 min pre-inject, $43{ }^{\circ} \mathrm{C}$, $3 \mathrm{~min}$; $45 \mathrm{~min}$ post-inject, $42{ }^{\circ} \mathrm{C}, 3 \mathrm{~min}$ ) while the control received no FUS. NIRF imaging used a Maestro EX (Perkin Elmer, MA, USA) with EX 704 $\mathrm{nm}$, EM 740-950 nm, $20 \mathrm{~nm}$ slicing, and unmixed against CF750-DSA. At $4 \mathrm{~h}$ the animals were sacrificed and tumours excised for gadolinium analysis by TXRF.

\section{Anti-tumour efficacy}

Two separate experiments were performed in single and double tumour mouse models. The single tumour study used three groups, all given injections i.v. tail vein: (i) control $(\mathrm{n}=5)$ which received only PBS; (ii) doxorubicin-HCL aq. ( $\mathrm{n}=5)$ at $4 \mathrm{mg} / \mathrm{kg}$; and (iii) iTSL-DOX $(\mathrm{n}=9)$ at equivalent to $4 \mathrm{mg} / \mathrm{kg}$ doxorubicin and two FUS treatments (Figure S3). NIRF imaging was used to track distribution and tumour uptake of the iTSL-DOX for $24 \mathrm{~h}$ postinjection. Tumour size and mouse body weights were recorded until they reached pre-determined endpoints ( $>10 \mathrm{~mm}$ longest axis or $>20 \%$ loss of prestudy weight) whereupon the animal was sacrificed. Tumour volume calculations were obtained using $\left(d^{2}\right.$ $\times D) / 2$ where $\mathrm{d}$ is the shortest tumour axis and $\mathrm{D}$ is the longest.

The double tumour study used two groups: (i) no-drug reference $(n=3)$ received only FUS on the right-side tumour, leaving the left to grow normally as a no-FUS control; and (ii) iTSL-DOX $(\mathrm{n}=10)$ at equivalent to $6 \mathrm{mg} / \mathrm{kg}$ doxorubicin and again FUS only on the right-side. Injections were given i.v. tail vein and FUS followed the same protocol. Tumour size and mouse body weights were recorded as before, and NIRF tracking of the iTSL was continued for 2 weeks post-treatment.

In vivo apoptosis assessment was carried out using the same double tumour model $(n=3)$ treated with iTSL-DOX but omitting CF750-DSA. AnnexinVivo 750 (100 $\mu \mathrm{L}$; Perkin Elmer) was administered $48 \mathrm{~h}$ after treatment and apoptosis assessed by NIRF imaging after $4 \mathrm{~h}$, according to the manufacturer's protocol. Tumours were then excised and fixed for H\&E staining.

\section{Murine tumour MRI}

For MRI, tumours were established and grown to $5-6 \mathrm{~mm} \varnothing$ as previously described $(\mathrm{n}=3)$. Mice were anesthetised and injected i.v. via the tail vein with iTSL $(200 \mu \mathrm{L}$; [Gd] $1.37 \mathrm{mg} / \mathrm{mL})$. MR imaging was performed beforehand and at $1 \mathrm{~h}, 3 \mathrm{~h}$ and $5 \mathrm{~h}$ afterwards. Animals were placed in the radiofrequency coil and flanked with a vial containing Gadovist ${ }^{\circledR}([\mathrm{Gd}] 3 \pm 0.01 \mathrm{mg} / \mathrm{L})$ as a reference before placement into the magnet bore and maintenance at $37^{\circ} \mathrm{C} . \mathrm{T}_{1}$-relaxometry and typical $\mathrm{T}_{1}$-weighted images were performed at $9.4 \mathrm{~T}$. $\mathrm{T}_{1}$-relaxometry was performed using a fast spin-echo sequence with the following parameters: TR 240, 400, 600, 800, 1000, 1200, 1500, and $3000 \mathrm{~ms}$; TE $11 \mathrm{~ms}$; matrix size $128 \times 128$; 1 scan; FOV $35 \times 35 \mathrm{~mm}$. Eight contiguous transverse $0.7 \mathrm{~mm}$ thick slices were collected, covering the tumour. Typical higher signal-to-noise $\mathrm{T}_{1-\text { weighted images were also obtained with the same }}$ sequence at TR $300 \mathrm{~ms}$; TE $7.765 \mathrm{~ms}$; 2 averages; FOV $35 \times 35 \mathrm{~mm}$, and matrix size $175 \times 175$.

$\mathrm{T}_{1}$ maps were generated as described above and matched ROI were identified in each slice for tumour, muscle (non-tumour control), and Gadovist ${ }^{\circledR}$ (positive control) in the adjacent-placed tube. Collated pixel intensities from these areas were combined for each animal and underwent frequency distribution analysis. To the resulting histograms, non-linear regression analysis was applied fitting to a Gaussian curve and the resulting best-fit mean and SD values cross-compared for each animal $(\mathrm{n}=3)$, time-point, and ROI. After ANOVA (1-way) significance analysis, these values were then combined across the subgroups to give overall distribution per subgroup. All statistical analysis was performed using Prism v 8.2.1 (Graphpad Software, San Diego CA, USA).

\section{Shelf-life stability}

The effects of short- and long-term storage on doxorubicin release were assessed using iTSL-DOX kept in a sterile vial at $\sim 5{ }^{\circ} \mathrm{C}$. The short-term study used samples warmed to room temperature for 10 $\min , 3 \mathrm{~h}$, or $24 \mathrm{~h}$ before incubation $\left(32-46^{\circ} \mathrm{C}\right.$; $\left.3 \mathrm{~min}\right)$ and fluorescence analysis. The long term study used samples taken directly from a $5{ }^{\circ} \mathrm{C}$ storage vial over 3 months and analysed for doxorubicin release, particle size, and polydispersity index.

\section{Results \& Discussion}

\section{Lipid synthesis and preparation of iTSL-DOX}

Two imaging-lipids were used in this study. Gd.DOTA.DSA (Scheme 1) is an MR contrast agent synthesised and characterised as previously described (Supplementary Material; Scheme S1) [21,25]. DOTAchelation was selected due to the reported efficiency of $\mathrm{Gd}^{3+}$ incorporation and high stability of the resulting complex. This is reported to significantly reduce transmetalation risk compared to classical 
diethylenetriamine pentaacetate derivatives [26]. After purification by liquid chromatography Gd.DOTA.DSA provided a $\sim 95 \mathrm{~mol} \%$ gadolinium loading as assessed using TXRF.

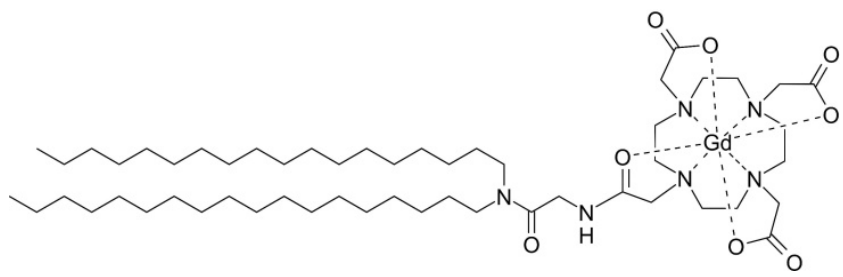

Scheme 1. Chemical structure of Gd.DOTA.DSA, a lipidic magnetic resonance imaging contrast agent.

iTSL were prepared with a Gd.DOTA.DSA composition of $30 \mathrm{~mol} \%$ (of total lipid) and underwent an iterative series of formulation optimisations to derive required physical and thermal release characteristics, while retaining a high labelling $\%$ for effective MR imaging. A second NIRF label (CF750.DSA) was included at $<0.1 \mathrm{~mol} \%$ and its presence did not appear to modify iTSL characteristics (save for the expected fluorescence). Doxorubicin loading was by incubation near the lipid membrane phase transition temperature, then purification by size exclusion chromatography. Each batch was characterised by DLS, DSC, HPLC, and TXRF with representative values: $\varnothing \mathrm{Z}_{\mathrm{avg}} 179 \pm 3 \mathrm{~nm}$, PDI $0.2 \pm$ $0.01 ; \zeta-3.2 \pm 0.1 \mathrm{mV}$; [Gd] $560 \pm 16 \mu \mathrm{g} / \mathrm{mL}$; [doxorubicin] $690 \pm 15 \mu \mathrm{g} / \mathrm{mL}$; drug/lipid ratio of $0.03 ; \mathrm{T}_{\mathrm{m}} 43.5^{\circ} \mathrm{C}$. These were considered in-line with our expectations, judging from previous experiences and reports from the literature.

\section{Thermally-induced doxorubicin release}

An effective TSL-drug needs to retain its cargo under normal in vivo conditions but rapidly release it on application of mild hyperthermia $\left(\sim 42{ }^{\circ} \mathrm{C}\right)$. Encapsulated doxorubicin has strongly self-quenched intrinsic fluorescence; this allows release from iTSL-DOX to be assessed by monitoring the intensity increase after incubation over a range of temperatures and times. Figure $1 \mathrm{~A}$ indicates little or no drug release on incubation at $37^{\circ} \mathrm{C}$ for $30 \mathrm{~min}$ in HEPES/glucose buffer. This was followed by a sudden increase of fluorescence intensity at temperatures $\geq 41{ }^{\circ} \mathrm{C}$, with more than $80 \%$ of the encapsulated drug released in 2 $\min$ at $43{ }^{\circ} \mathrm{C}$. iTSL-DOX demonstrated almost identical release profiles in buffer and serum-like conditions (Figure 1B) which bodes well for its use in vivo [27].
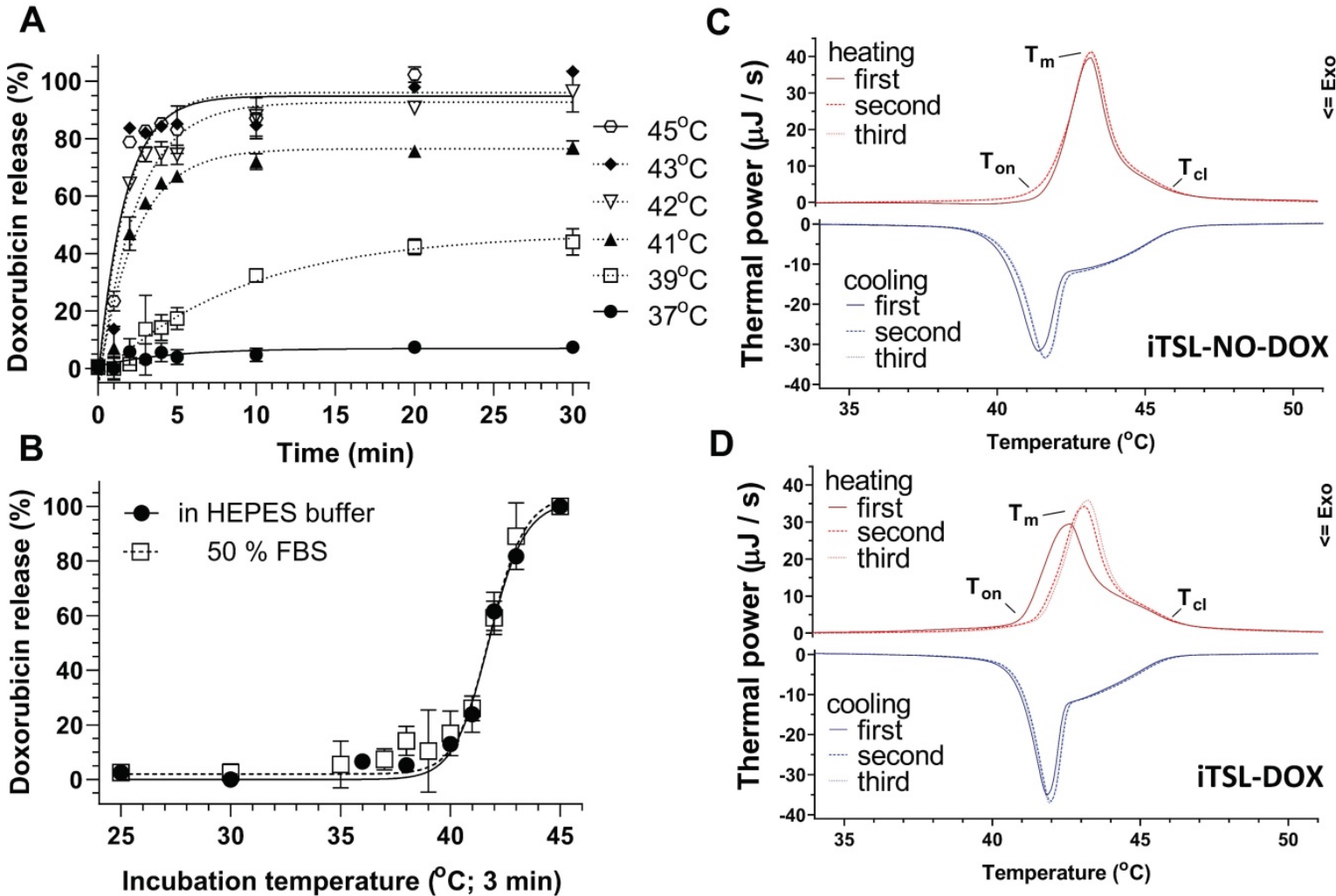

Figure 1. iTSL-DOX characterisation: (A) Doxorubicin release from iTSL at various temperatures in HEPES; (B) for 3 min at various temperatures in comparison with buffer containing $50 \%(\mathrm{v} / \mathrm{v})$ fetal bovine serum (FBS). Release is monitored by the increase of intrinsic doxorubicin fluorescence (Ex480/Em590 nm) as it leaves the self-quenched encapsulated state $(n=3$; mean \pm SD); (C) Liquid-phase differential scanning calorimetry of iTSL without and; (D) with encapsulated doxorubicin. Each study consisted of $3 x$ sequential heating-cooling rounds from $25-70{ }^{\circ} \mathrm{C}$ at $1{ }^{\circ} \mathrm{C} / \mathrm{min}$. iTSL were in HEPES/glucose buffer and used the same as a thermal reference. Indicative onset $\left(\mathrm{T}\right.$ on), melting $\left(\mathrm{T}_{\mathrm{m}}\right.$ ), and closure temperatures ( $T_{\mathrm{cl}}$ ) for each heating thermograph are: iTSL-NO-DOX: (first) $\mathrm{T}_{\text {on }} 41.2, \mathrm{~T}_{\mathrm{m}} 43.3, \mathrm{~T}_{\mathrm{cl}} 45.8$, (second) $\mathrm{T}_{\text {on }} 41.1$, $\mathrm{T}_{\mathrm{m}} 43.3$, $\mathrm{T}_{\mathrm{cl}} 46.0$; iTSL-DOX: (first) $\mathrm{T}_{\text {on }} 40.3, \mathrm{~T}_{\mathrm{m}} 42.7, \mathrm{~T}_{\mathrm{cl}} 46.0$, (second) $\mathrm{T}_{\text {on }} 40.9, \mathrm{~T}_{\mathrm{m}} 43.2, \mathrm{~T}_{\mathrm{cl}} 46.0$; all $\pm 0.2{ }^{\circ} \mathrm{C}$. $\mathrm{T}_{\text {on/cl }}$ were calculated as the first and last temperatures at which the thermal power was $5 \%$ of $\mathrm{T}_{\mathrm{m}}$. 

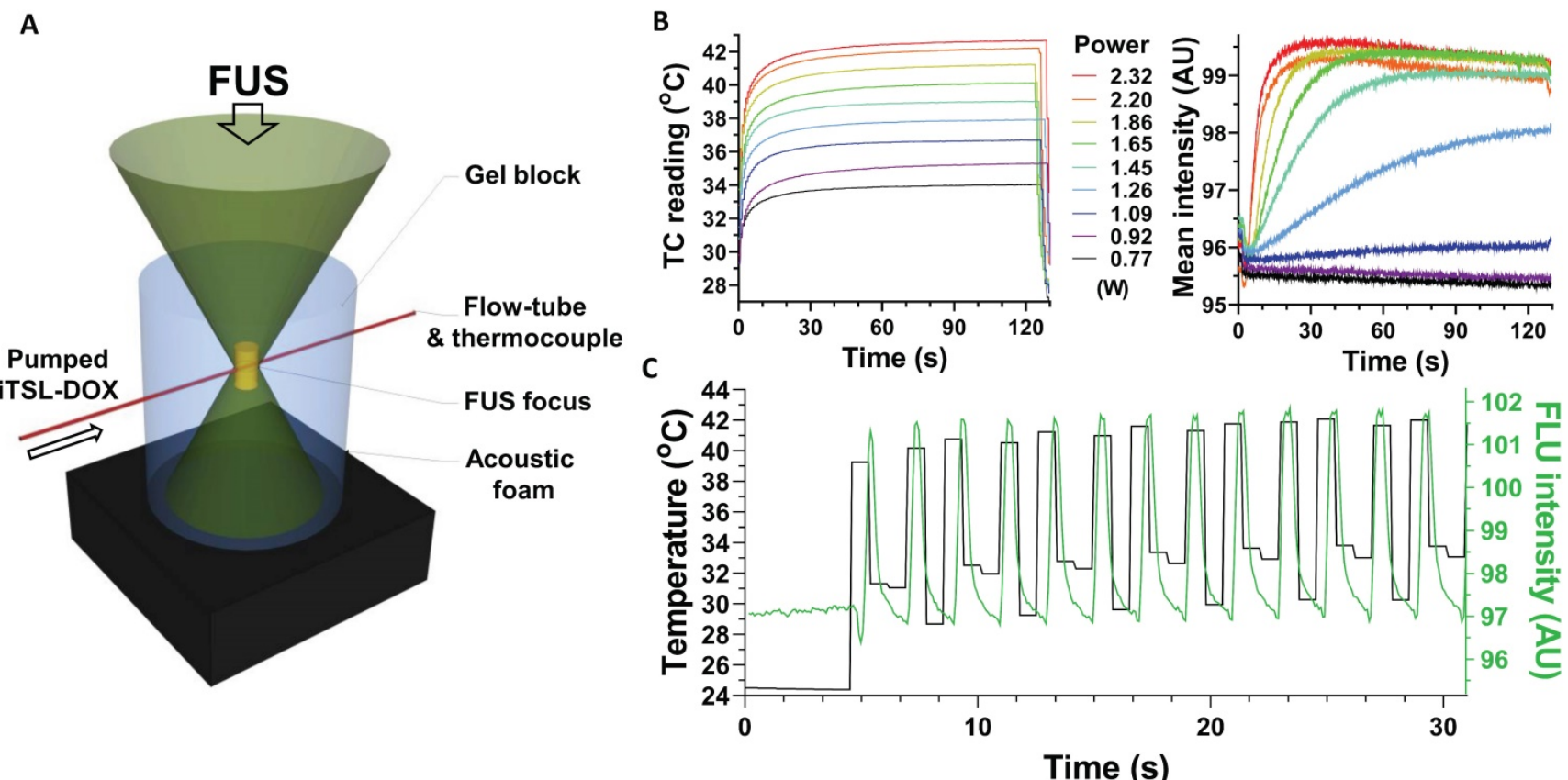

Figure 2. Assessing doxorubicin release under FUS, measured by intrinsic doxorubicin fluorescence; (A) schematic showing a polyacrylamide gel embedded flow-tube below a focused ultrasound transducer (Figure S1); (B) graphs of thermocouple (left) and fluorescence intensity (right) readings seen with increasing power levels of constantly applied FUS, using a fresh bolus of iTSL-DOX for each 2 min insonation; (C) Fluorescence intensity and temperature plotted against time under pulsed FUS and constant iTSL-DOX flow.

This thermally-induced drug release aligns well with liquid-phase DSC thermograms collected for iTSL-NO-DOX (without loaded doxorubicin) and iTSL-DOX (Figure 1C-D). DSC investigates the changes in bulk heat capacity on heating or cooling from $25-70{ }^{\circ} \mathrm{C}\left(1{ }^{\circ} \mathrm{C} / \mathrm{min}\right.$; $3 \times$ sequential heat-cool cycles). The resulting peaks/troughs are mainly due to temperature-driven phase changes in the lipid membrane; these are associated with pore formation and release of the encapsulated drug. iTSL-NO-DOX and iTSL-DOX have slightly different first heating thermograms, with the latter showing a $1{ }^{\circ} \mathrm{C}$ down-shift in the peak leading edge to $\sim 41.5{ }^{\circ} \mathrm{C}$ (Figure 1D). This initiation temperature matches the doxorubicin release point seen from the fluorescence studies. This strongly suggests that DSC is able to detect the release of encapsulated doxorubicin during this first round of heating. Subsequent cycles are then the same as iTSL-NO-DOX, likely because the drug release is complete. Overall, doxorubicin release parameters suggest that iTSL-DOX formulation has been successfully optimised to adjust for the high $\mathrm{mol} \%$ of MRI CA lipid [28,29].

TSL tend to be unstable when incubated in serum [30] and this has previously caused difficulties during clinical development. For example, a widely used LTSL (lysolipid-based temperature sensitive liposome) formulation released $50 \%$ doxorubicin after $30 \mathrm{~min}$ in $20 \mathrm{v} \%$ serum [31]. This was attributed to the presence of adhered plasma proteins causing $~ 70 \%$ of the lysolipid to dissociate from the liposomal membrane within the first hour. This likely resulted in significant drug leakage in the blood stream [32,33]. We investigated doxorubicin release from iTSL-DOX incubated at $37^{\circ} \mathrm{C}$ in buffer and in $50 \mathrm{v} \%$ foetal bovine serum. After $60 \mathrm{~min}$ incubation there was minimum leakage $(\sim 5 \%)$ doxorubicin into buffer but $30 \%$ into serum (Figure S4). This was a significant improvement over previously reported LTSL and suggested that doxorubicin leakage in blood circulation should be greatly reduced.

\section{FUS-induced doxorubicin release}

Incubation studies at different temperatures were a facile method to assess iTSL-DOX thermossensitivity but did not demonstrate drug release under FUS insonation. Here, we investigated FUSinduced doxorubicin release in real time using a custom made flow-tube setup (Figure 2A). A solution of iTSL-DOX was pumped through a capillary tube embedded in a block of tissue mimicking gel [34]. FUS was applied using a small-animal transducer array and doxorubicin release was monitored using fluorescence imaging with a video camera, while a thermocouple tracked the temperature near the FUS focus (Figure 2, Figure S1).

Initial studies used the tube as a refillable sample cell, applying a particular degree of FUS acoustic power for $2 \mathrm{~min}$, then washing out, replacing with a fresh aliquot of iTSL-DOX, and repeating at a higher power. As expected, the focus peak temperature increased directly with applied FUS power (Figure 2B 
left) as did the doxorubicin fluorescence (Figure 2B right). The latter also showed release profiles similar to those from the thermal-induced release studies, with doxorubicin release rates dependent on the acoustic power applied.

To investigate the doxorubicin release responsiveness, the setup was adjusted to have a constant flow of fresh iTSL-DOX through the tube (modelling blood capillaries) while applying pulsed FUS at a power level sufficient to rapidly raise the focus temperature to our expected in vivo target of $\sim 41$ ${ }^{\circ} \mathrm{C}$. Doxorubicin fluorescence then showed a rapid and temporally coherent increase that matched the measured temperature spikes (Figure 2C). Acoustic power is an important parameter that can control the rate of the release and (with this system) at $2.32 \mathrm{~W}$ the drug release appears effectively instantaneous. This implies that spatiotemporal control of the application of the acoustic power can lead to precise remote control of the drug released.

\section{In vitro $M R$ relaxivity}

MR contrast agents (CA) are used to increase the imaging contrast between tissues, usually by shortening the longitudinal $\left(\mathrm{T}_{1}\right)$ relaxation time or the transverse $\left(T_{2}\right)$ relaxation time. There are two main approaches to developing liposomes that enhance tumour MR $\mathrm{T}_{1}$ contrast: (i) encapsulation of small molecule $\mathrm{Gd}^{3+}$-chelates into the aqueous core, which then label the tumour after thermal release; or (ii) the attachment of anchored $\mathrm{Gd}^{3+}$-chelates to the lipid membrane. The latter allows for improved water accessibility and better contrast since the interaction of $\mathrm{Gd}^{3+}$ with the surrounding water protons is not inhibited by the lipid membrane [35].

Figure $3 \mathrm{~A}$ presents $\mathrm{T}_{1}$-weighted images of phantoms containing different dilutions of iTSL and Gadovist ${ }^{\circledR}$ (a positive control) at comparable molar concentrations of $\mathrm{Gd}^{3+}$ and at a magnetic field strength of $9.4 \mathrm{~T}$. iTSL showed enhanced signal intensity dependent on the concentration, similar to Gadovist ${ }^{\circledR}$ serial dilutions. Figure 3B-C shows the molar $\mathrm{T}_{1}$ relaxivities of iTSL and Gadovist ${ }^{\circledR}$ at 9.4 and $7 \mathrm{~T}$. This is a measure of the efficacy of the CA to decrease the $T_{1}$ relaxation time of water protons in its surrounding. The greater the $r_{1}$, the smaller the amount of the CA is needed to achieve the same $T_{1}$ decrease and hence brightness enhancement in the $\mathrm{T}_{1}$-weighted $\mathrm{MR}$ image [36]. At 9.4 $\mathrm{T}$ iTSL shows lower efficiency as a $\mathrm{T}_{1}$-CA compared to the Gadovist ${ }^{\circledR}$ - one of the most efficient small-molecule cyclic-gadolinium CA used in clinic, however magnet field strength affects relaxivity of contrast agents [37]. The relaxivity of Gd-based liposomal CA is generally higher when the common clinical magnets (1.5 and
3T) are used. At $7 \mathrm{~T}$, the molar relaxivities of iTSLDOX and Gadovist ${ }^{\circledR}$ were similar and pre-heating to release the doxorubicin gave a slight improvement. We judge this is likely due to a change of water permeability across the iTSL membrane. Increased membrane porosity improves water accessibility to the liposome core, allowing the normally isolated inner surface Gd.DOTA.DSA to have a contrast enhancing effect. Under either condition, iTSL appeared to be an effective $\mathrm{T}_{1}$-CA.

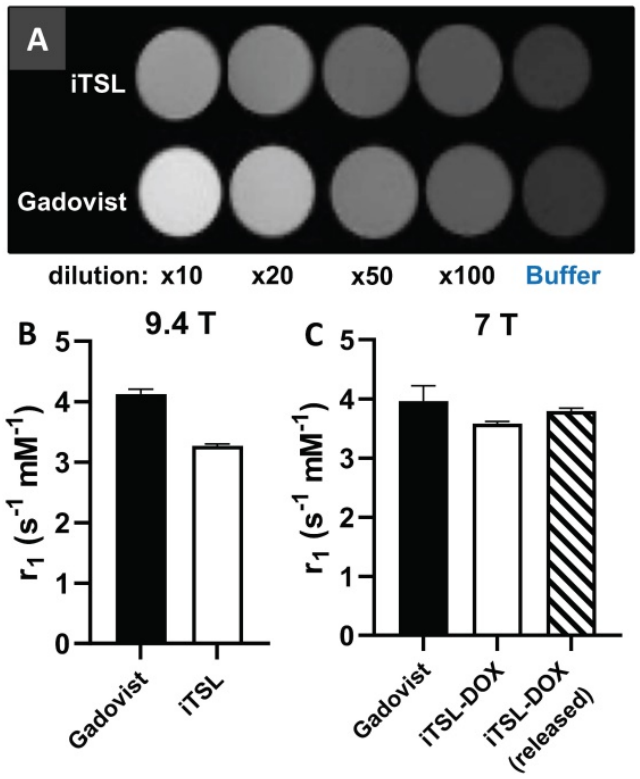

Figure 3. MRI $T_{1}$ relaxivity measurement of iTSL and Gadovist ${ }^{\circledR}$ (positive control) in phantoms; (A) $9.4 \mathrm{~T} \mathrm{MRI}$ images of samples with approximately matched gadolinium content (initially $1.46 \mathrm{mg} / \mathrm{mL}$ for Gadovist $(1.36 \mathrm{mg} / \mathrm{mL}$ for $\mathrm{iTSL}$ ) using HEPES/glucose buffer as a baseline and for dilution; (B) Molar relaxivities ( $\left.r_{1}\right)$ were calculated from the relaxation rate change as a function of gadolinium concentration (as measured by TXRF). A higher $r_{1}$ implies a stronger contrast agent; (C) Equivalent $r_{1}$ values for doxorubicin loaded iTSL using $7 \mathrm{~T}$ MRI, with initial gadolinium concentrations: $0.43 \mathrm{mg} / \mathrm{mL}$ Gadovist ${ }^{\circ} ; 0.26 \mathrm{mg} / \mathrm{mL}$ iTSL and again using HEPES/glucose buffer for dilution. The iTSL-DOX (released) sample was previously incubated for $3 \mathrm{~min}$ at $45^{\circ} \mathrm{C}$ to thermally activate the liposomes.

\section{Shelf-life stability}

There are only a few published studies that investigate how long-term storage affects TSL characteristics. TSL formulations contain lyso-lipids and may show limited colloidal or drug-retention stability (even at reduced temperature). This can be a particular issue at lab scale production where the methods of preparation, sterility assurance, and storage conditions are likely to be sub-optimal.

To inform on this, we introduced a stability study to investigate the effect of short- and long-term storage on the integrity of aseptically produced iTSL-DOX (Figure S5). Samples were initially left at room temperature for 3 or $24 \mathrm{~h}$ - durations selected to mimic the time range that a formulation might be left on a bench during a human trial or large animal study. iTSL-DOX showed only minimal changes to 
the doxorubicin release profile after $24 \mathrm{~h}$ and none at 3 $\mathrm{h}$, indicating a robust formulation that retains its properties for a reasonable time outside of a fridge. Long-term stability was then assessed using samples taken at intervals from iTSL-DOX in cold-storage $(\sim 5$ $\left.{ }^{\circ} \mathrm{C}\right)$ for up to 3 months. Again there was no apparent change to the release profile as the liposome ages, confirming the formulation robustness. DLS analysis of the same samples also showed no significant changes to average nanoparticle diameter or the PDI. We conclude that iTSL-DOX has good storage robustness, which obviates the requirement for fresh preparations between rounds of in vivo studies.

\section{Avoidance of gadolinium toxicity}

Free $\mathrm{Gd}^{3+}$ cations are toxic due to interference with $\mathrm{Ca}^{2+}$-dependant biochemical processes, amongst other effects [38]. This toxicity is greatly reduced when the metal is chelated but there are currently concerns due to the demonstrated links between the use of Gd-based MRI contrast agents and rare but serious incidents of nephrogenic systemic fibrosis in patients with poor kidney function [39]. Therefore, there is a question as to the safety of gadolinium contrast agents that may 'leak' $\mathrm{Gd}^{3+}$ due to chelation competition from biomolecules in the body [40].

We investigated the integrity of iTSL's $\mathrm{Gd}^{3+}$ chelation under challenging conditions. Figure S6 shows a log plot of the leakage of $\mathrm{Gd}^{3+}$ (as assessed by TXRF) through dialysis membranes against water or serum, over two days. This is compared to the behaviour of matched concentration controls of unchelated $\mathrm{Gd}^{3+}$ aq. from gadolinium standards. The pore size of the dialysis cassette $(10 \mathrm{kD}$ MWCO) blocked intact iTSL but allowed the passage of 'free' (or small molecule bound) $\mathrm{Gd}^{3+}$. Hence gadolinium assayed from the external chamber was either from the control or was initially part of Gd.DOTA.DSA and subsequently extracted by competition from the medium. FBS was assessed as serum contains many of the proteins and ions found in blood and provides a

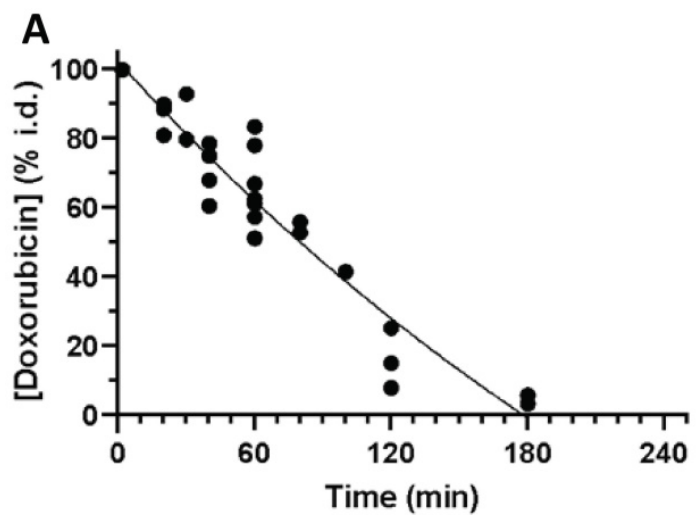

fairly realistic approximation of the conditions that Gd.DOTA.DSA would encounter once injected i.v.

It was observed that while the 'free' $\mathrm{Gd}^{3+}$ controls diffusion equilibrated, there was very little metal detected from the iTSL and no obvious increasing trend over the $48 \mathrm{~h}$ study. This is in-line with previous reports which demonstrated greatly reduced $\mathrm{Gd}^{3+}$ leakage from the macrocyclic chelators under serum conditions compared to linear chelators [38,39]. We concluded that iTSL was safe for i.v. administration and further clinical development.

\section{Blood clearance profiles in mice}

To develop a better understanding of the pharmacokinetic behaviour of iTSL-DOX, we used LC-MS/MS and TXRF analysis to assess the blood clearance rates of doxorubicin and Gd.DOTA.DSA (Figure 4). The iTSL-DOX was injected i.v. and blood samples were taken at predetermined time points. Doxorubicin (whether encapsulated or not) appears to be cleared from the blood stream by $3 \mathrm{~h}$, with a $\mathrm{t}_{1 / 2}$ of $\sim 80 \mathrm{~min}$. This assay detected both iTSL encapsulated doxorubicin and 'free' drug. In comparison with previously presented data on similar TSL-DOX formulations, ours indicate a slightly shorter half-life but are in general agreement with the short term kinetics of thermosensitive liposomes in blood. Blood circulation stability is a concern, as previously reported TSL-DOX has demonstrated a tendency to leak doxorubicin - a factor that may have significantly limited its clinical success. We also measured the liposome kinetics by assessing blood gadolinium levels. It is evident that while doxorubicin circulates in blood up to $3 \mathrm{~h}$ post-administration, Gd.DOTA.DSA is detectable for much longer. Considering the slow doxorubicin leakage under blood-like conditions (Figure S4) it seems reasonable that past $3 \mathrm{~h}$ some iTSL are still circulating but are empty of doxorubicin and Gd.DOTA.DSA eliminates from the blood with a $t_{1 / 2}$ of $\sim 150 \mathrm{~min}$.

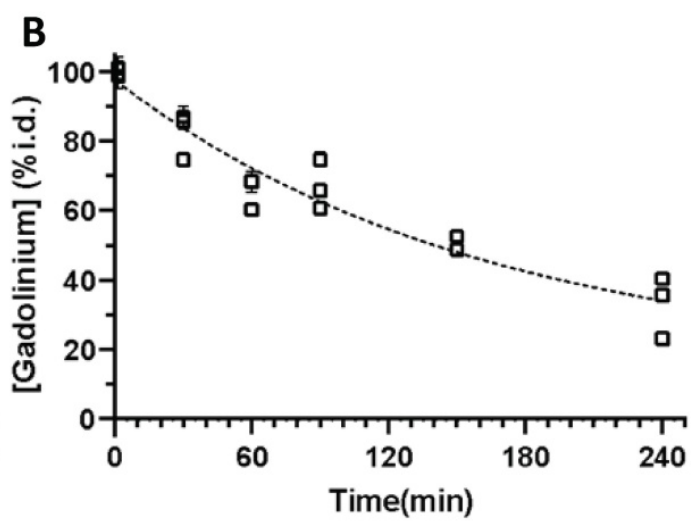

Figure 4. Clearance of iTSL-DOX; (A) doxorubicin $(n=7)$ and; $(\mathbf{B})$ gadolinium $(n=6)$ from mouse blood circulation, shown as \% of injected dose. Mice were injected (i.v. tail) with iTSL-DOX (4 mg/kg equivalent doxorubicin), then blood samples were collected at time points. Values are the mean of $2-3$ repeat analyses per sample \pm SEM. 

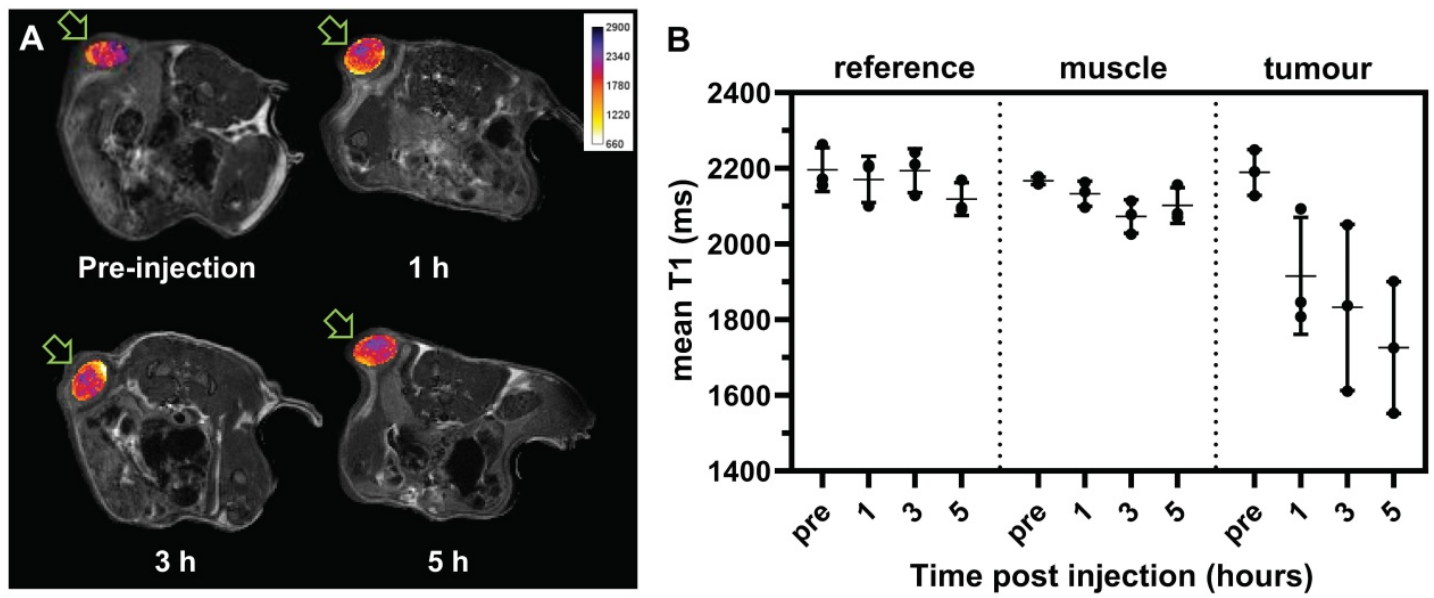

Figure 5. Spin-echo transverse $\mathbf{T}_{1}$ weighted MR imaging, iTSL (1.37 mg/mL gadolinium) was injected (i.v. tail vein) to mice, (A) Imaging (9.4 T) of the same representative animal pre-injection, then $1 \mathrm{~h}, 3 \mathrm{~h}$, and $5 \mathrm{~h}$ post-injection. $\mathrm{T}_{1}$-weighted tumours images are overlaid with $\mathrm{T}_{1}$ relaxivity maps (in false colour) for the region-of-interest (ROI), as indicated with arrows; (B) Comparative $T_{\text {I }}$ values for selected volumes-of-interest (combining the ROls from matched slices) from a single-tumour mouse study ( $\mathrm{n}=3$; individual values $\cdot$, mean as horizontal bar, SD as vertical bar); see also Figure S7. A lower $\mathrm{T}_{1}$ implies a stronger contrast enhancement.

TSL formulations are generally designed to avoid the very long blood circulation times usually observed with liposomes like Doxil®. They are intended to be combined with hyperthermia and to efficiently deliver a drug into the tumour interstitial and/or intravascular space. To achieve this regional delivery TSL should be activated while they circulate at high concentrations and while they retain their drug cargo. This indicates that the triggered drug release should occur before the normal blood clearance $\mathrm{t}_{1 / 2}$ time point of the drug delivered by TSL. This is the suggested time window for maximal tumour drug concentration once a thermal trigger is applied [32]. Shorter half-lives can have the additional advantage of diminishing exposure of off-target tissues to doxorubicin. This may have a significant advantage of reducing doxorubicin-related cardiotoxicity, as reported for Doxil ${ }^{\circledR}$ [43]. But at this point there are no studies showing a comparison of the therapeutic index between Doxil ${ }^{\circledR}$ and TSL-DOX.

\section{$T_{1}$-weighted MRI of iTSL in tumour bearing mice}

While MRI phantoms show that iTSL has good $\mathrm{T}_{1}$-relaxivity, it is essential to demonstrate that they can also be detected in vivo. Figure 5A shows typical $\mathrm{T}_{1}$-weighted images of subcutaneously implanted tumours in mice before and after iTSL administration. Similar studies reported by Liu et al. used the relative signal intensity from $\mathrm{T}_{1}$-weighted images of kidney and liver to study the kinetics of Gd.DTPA labelled liposomes [44]. In our experiment, i.v. injection of iTSL resulted in an increased MR signal intensity in the tumour within an hour. Figure $5 \mathrm{~B}$ shows the changes in mean $T_{1}$ of tumour volumes-of-interest (combining ROI across multiple imaging slices) compared to a reference vial of Gadovist ${ }^{\circledR} \mathrm{CA}$ and a reference tissue (skeletal muscle). The $\mathrm{T}_{1}$ of reference regions were not expected to change significantly over time but tumours showed decreased $T_{1}$ due to the accumulation of iTSL and this continued until the end of the study. An increase in the variability of the $T_{1}$ values across the tumour were also observed, which was attributed to high tissue heterogeneity (due to the developing necrotic core) and differential iTSL uptake depends on the amount of blood perfusion. A histogram analysis approach was used to better distinguish the changes under these heterogeneous conditions (Figure S7).

The observed reductions in mean $T_{1}$ demonstrated the accumulation of iTSL in the tumour soon after injection and its persistence for at least $5 \mathrm{~h}$ post-injection. This finding supports the hypothesis that iTSL can be tracked post-injection for spatiotemporal control of the FUS triggered release. For a radiologist working with MRgFUS a change in the MR signal from the tumour could indicate the optimal time for application of FUS. It could also confirm that the treatment was successful, as there would likely to be an enhanced tumour signal post treatment.

\section{Design of the FUS protocol}

iTSL-DOX pharmacokinetics information directs the choice of FUS parameters, in particular the timing respective to liposome administration. The blood clearance results showed that the remaining iTSLencapsulated doxorubicin is likely to be significantly reduced after $1 \mathrm{~h}$ and effectively eliminated after $3 \mathrm{~h}$. This suggests that FUS should be applied soon after injection to best affect doxorubicin release from iTSL that are still loaded with drug. However, this needs to be balanced by allowing sufficient time for iTSL-DOX to accumulate into the tumour. The use of multiple 
rounds of FUS could trigger release from iTSL-DOX while still being infused, similar to the release observed in the in vitro system in Figure 2. Our previous imaging results with a similar iTSLtopotecan formulation demonstrated that the drug is rapidly released on application of FUS and that treatment repetition can maximise the available drug in the tumour [21]. Short rounds of FUS were also found to be equally efficient compared to prolonged continuous hyperthermia in a recent study [45]. We concluded that brief ( $\leq 5 \mathrm{~min})$ periods of FUS hyperthermia $\left(\leq 43{ }^{\circ} \mathrm{C}\right)$ would be sufficient to induce drug release (intravascular and/or interstitial). In our experience brief FUS treatments are also safer than longer ones, being less stressful for the animals, reducing the risk of skin/tumour burns or other over-heating related damage, and (importantly) avoiding false positive results (e.g. from hyperthermic ablation, leading to tumour tissue damage masking the effect of the released chemotherapeutic).

Application of FUS before drug injection has been reported to improve therapeutic efficacy. For example, ThermoDox ${ }^{\circledR}$ preclinical development information strongly suggests that the heating of the tumour needs to precede injection of the TSL [8]. Other studies have demonstrated effective treatment with FUS applied just before and then during infusion [46], or immediately after injection [47]. In addition, the phase-III HEAT clinical trial computer modelling led to application of radiofrequency ablation $15 \mathrm{~min}$ post-infusion [48] and the TARDOX study indicated application of FUS shortly after infusion [20]. The high variability of TSL FUS protocols across the literature suggests that better feedback tools are required to allow the identification of optimum timing. Feedback through imaging of the TSL during treatment can make the process easier for the radiologists. For instance, FUS could be applied once MRI contrast has reached a predefined level (contrast) in the tumour. Under clinical conditions FUS-induced hyperthermia would be applied and controlled using concurrent imaging (MRgFUS) which should identify the tumour and margins but would also be used to monitor the tissue temperature, iTSL accumulation and provide a confirmation that appropriate dose has reached the tumour [49].

\section{NIRF imaging of iTSL+FUS treated mice}

Biodistribution studies were carried out by administering NIRF-labelled iTSL-DOX into mice with human TNBC (MDA-MB-231) cell tumours. These were placed on the haunch to allow easier and safer access of the FUS beam and were $\sim 5 \mathrm{~mm}$ diameter on treatment day. Two groups received iTSL-DOX but only the one underwent FUS-induced hyperthermia. Here we combined one brief sonication before injection with a second round after but with enough delay to allow enhanced iTSL uptake into the tumour. NIRF tracking of iTSL uptake allowed us to select a $45 \mathrm{~min}$ post-injection time point for the second FUS, leading to significant tumour accumulation while still corresponding to $\sim 70 \%$ availability of injected dose of doxorubicin in the blood stream (Figure 4). At this point it is likely that iTSL-DOX still encapsulates the great majority of the doxorubicin since both doxorubicin and gadolinium are found at similar \% of injected dose.

In all experiments the FUS focal volume was deliberately placed slightly above and outside the targeted tumour. This was done to avoid the formation of a 'hot spot' within the tumour body, ensuring the effects seen were due to FUS-induced mild hyperthermia and not ablative tissue damage. To control the applied FUS power, tumour temperatures were monitored using fine-wire thermocouples that were implanted around the tumour (Figure 6). This was to substitute for the more elaborate MR thermal maps provided by clinical MR-guided FUS equipment
A

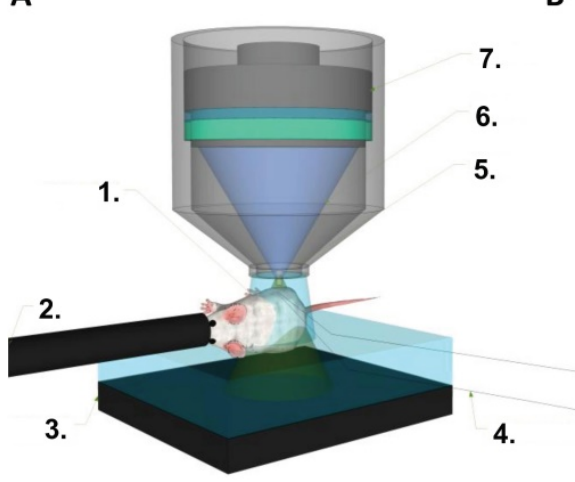

B

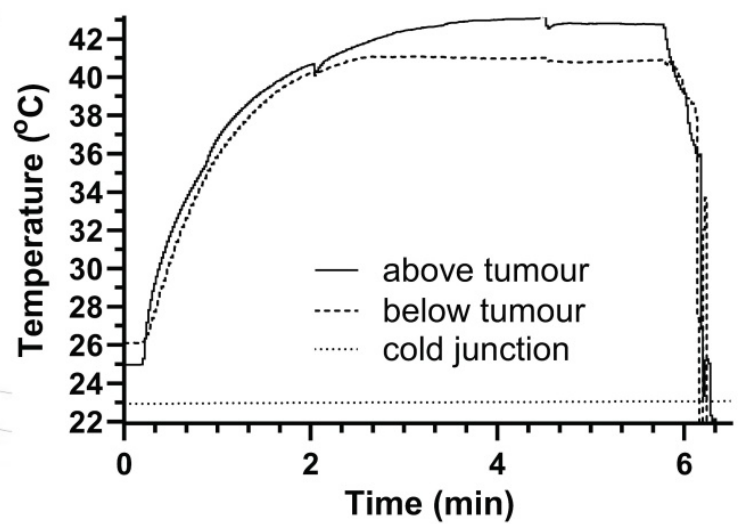

Figure 6. Preclinical FUS studies. (A) Mouse focused ultrasound showing key components: 1. degassed gel; 2. anaesthetic; 3 . acoustic foam; 4 . thermocouples; 5 . focus; 6 . ultrasound biconic; 7. transducer; (B) Tumour temperatures measured using fine-wire thermocouples implanted s.c. above and below the tumour (with respect to the transducer focus location). The cold junction is in the recording electronics and $\sim 2 \circ \mathrm{C}$ above RT. 
[50]. Thermocouples were inserted below and above the tumour, avoiding the FUS focal volume, and temperatures were monitored throughout the treatment. Figure 6B provides typical thermographs achieved during an FUS treatment showing that temperatures reach a constant plateau.

NIRF imaging allows monitoring of the level of iTSL within the tumour compared to the rest of the body [46]. We selected it as a semi-quantitative but robust method that was expected to significantly facilitate clinical translation [51]. Figure 7 shows representative mice from groups that have been administered iTSL-DOX either with or without FUS and then undergone real-time tracking by NIRF imaging. It is evident that the absence of FUS led to little accumulation of iTSL in the tumour. In contrast, brief FUS treatments caused a substantial increase in tumour-localised signal. We monitored mice up to $4 \mathrm{~h}$, at which point the remnant circulating iTSL are likely to be empty and the rate of their partition in the tumour compartment should be decreasing [51]. We also TXRF analysed tumour samples for gadolinium content at $4 \mathrm{~h}$ (Figure 7B). Given the chelation stability of Gd.DOTA.DSA this should be a reasonable indicator for the amount of iTSL that has been retained in tumours at this time point. At $4 \mathrm{~h}$ post injection, FUS-treated tumours levels were more than double that of the untreated group.

\section{Doxorubicin release and iTSL clearance}

We also assessed iTSL-DOX + FUS treatment on animals bearing two tumours (one on each side). In this double-tumour model, the left side received no FUS whilst the right side was treated as before. This allows FUS effects to be assessed more directly as each animal also acts as control. Figure 8 presents real-time
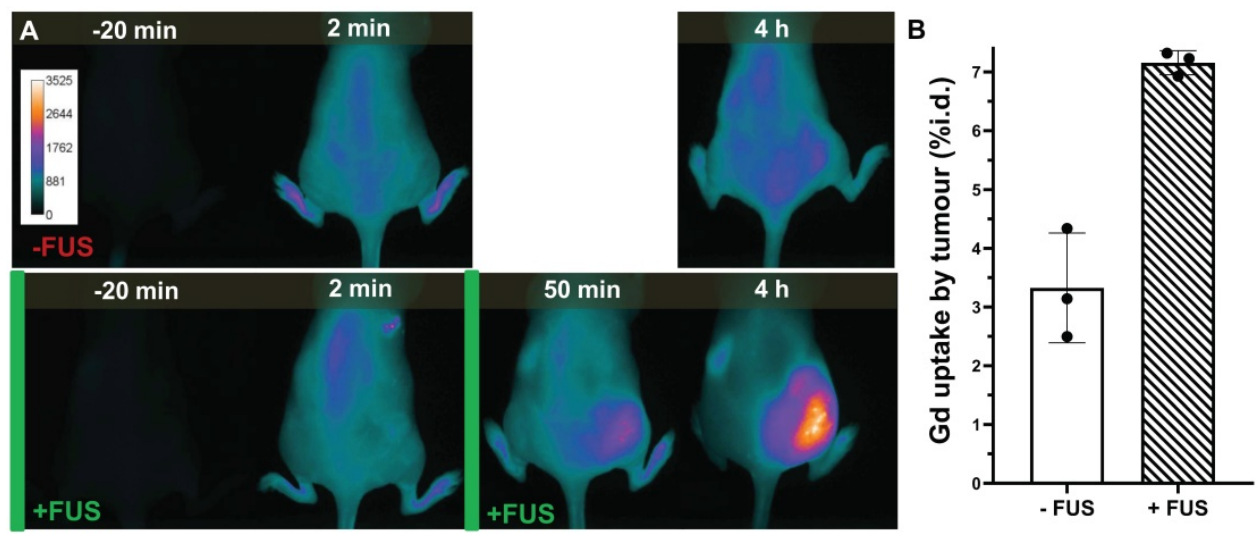

Figure 7. Representative imaging of iTSL-DOX uptake; (A) Near-infrared fluorescence imaging comparison of mice injected (i.v. tail; $t=0)$ with iTSL-DOX, and without (-FUS) or with (+FUS) focused ultrasound on the right side. Images are shown pre-, then post-injection and the FUS treatments are indicated by the green bars. The CF750-DSA liposome label was excited at $704 \mathrm{~nm}$ and fluorescence emission collected over 740-950 nm. (B) Gadolinium tumour concentration ( $\mathrm{n}=3$; mean \pm SD; normalised by tumour mass) assessed by TXRF.

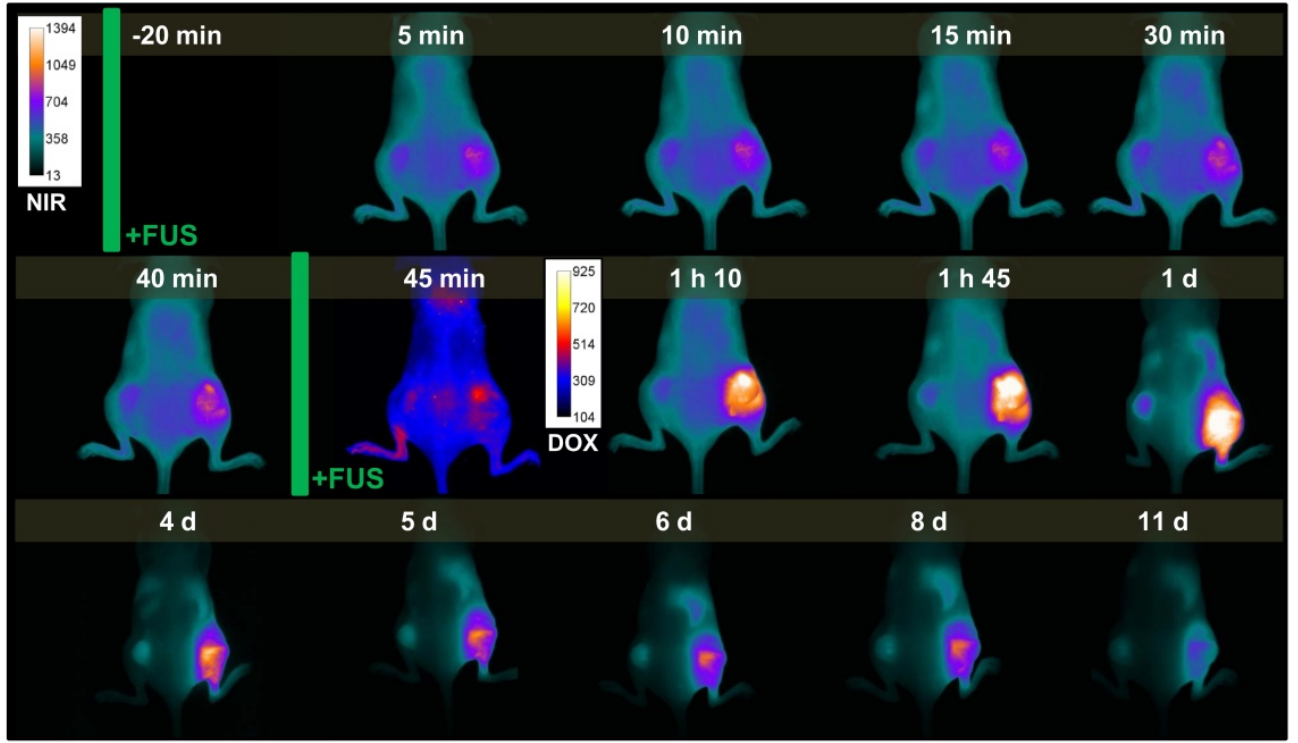

Figure 8. Representative example of near-infrared (NIRF) and doxorubicin fluorescence imaging during the dual-tumour study. Time points are pre/post-injection and focused ultrasound (FUS) treatments are indicated by green bars. Right tumour received FUS, while left tumour did not receive FUS. NIRF images are coloured cyan-yellow, doxorubicin are indigo-red. 
NIRF imaging of iTSL-DOX kinetics on a mouse bearing double MDA-MB-231 tumours and with the optimised FUS protocol applied on the right-hand side. It is evident that FUS-treated tumour allowed accumulation to a much larger extent compared to the FUS-untreated tumour. It is also evident that intrinsic doxorubicin fluorescence (shown here in red against blue) is distinguishable in the +FUS tumour to larger extent compared to the untreated and only when the second round of hyperthermia was applied.

Figure 8 also shows that the CF750.DSA lipid appears to clear slowly from the treated tumour for up to 2 weeks post-administration. The signal from the untreated tumour appears to fade more rapidly. It is possible that FUS enhances the permeation and incorporation of liposome components in the tumour. The concept of FUS-enhanced delivery of lipophilic drugs transferred this way has not yet been explored.

\section{Efficacy of iTSL-DOX + FUS}

Once the FUS protocol had been demonstrated to be effective at increasing iTSL-DOX uptake, we expanded to an efficacy study using a single (right-side) TNBC tumour model and groups: (i) nil treatment; (ii) doxorubicin alone; and (iii) iTSL-DOX with FUS. Doxorubicin dosages were matched at 4 $\mathrm{mg} / \mathrm{kg}$ and the FUS, imaging, and size/weight measurement timings were as described in Figure S3.
Figure 9A presents the tumour growth effects of iTSL-DOX in combination with the brief FUS treatment protocol, compared to controls of doxorubicin or nil-drug (both without FUS). We can observe that FUS in combination with iTSL-DOX induces a powerful retardation in TNBC tumour growth. By contrast, little effect is seen in the group treated with dose-matched doxorubicin alone. Figure 9B shows changes of body weight, with the mice initially at $\sim 22$ g. Both drug-treated groups showed weight loss, with the doxorubicin-only mice showing gradual but continual weight reduction over 2 weeks post-injection. Mice treated with iTSL-DOX+FUS initially lost $\sim 10 \%$ (Home Office license limit being $20 \%$ of initial body weight) but then stabilised and appeared to partially recover. Overall, mice that were treated with iTSL-DOX+FUS responded better to treatment and survived longer as seen by the Kaplan-Meir curve at Figure 9C.

This breast cancer tumour model was previously used with TSL-DOX and Lokerse et al. reports similar levels of tumour growth inhibition at higher dose of 5 $\mathrm{mg} / \mathrm{kg}$ doxorubicin [52]. However, in Lokerse's study hyperthermia (non-focused, the authors did not apply FUS) was applied for a substantially longer time of 60 min. We achieved similar effects with $1 / 10^{\text {th }}$ of the hyperthermia application time and in a focused manner. Clinical MR-imaging could be of significant

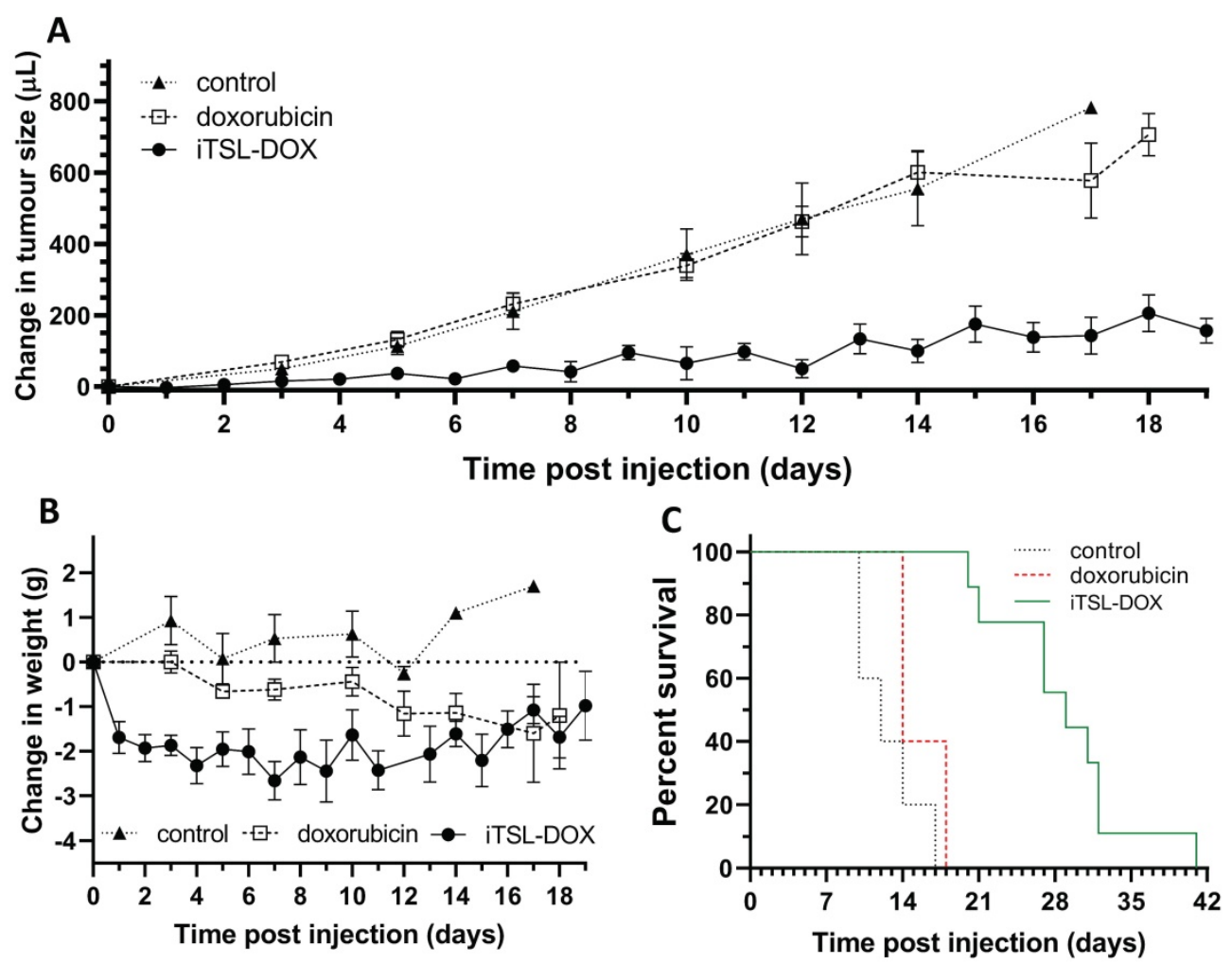

Figure 9. Single-tumour mouse studies, showing $(\mathbf{A})$ averaged tumour volumes (mean \& 1 SEM) for the control ( $\mathrm{nil}$ drug; $\mathrm{n}=5$ ), doxorubicin $(\mathrm{n}=5$ ), and iTSL-DOX treated $(n=9)$ groups; (B) body weights and; (C) Kaplan-Meier plots for the same, showing survival. Dosage was $4 \mathrm{mg} / \mathrm{kg}$ equivalent of doxorubicin and focused ultrasound was applied before/after injection. 
assistance here, providing information for location and required thermal dose. A related concern is that extended FUS treatments are harder to translate to the clinic, requiring a patient to spend a significant time immobilised in the scanner [45]. Apart from the discomfort, this has important implications on heating precision. Precise hyperthermia for deep seated tumours requires minimised target motion, suggesting that brief repeated rounds may be more practical than $1 \mathrm{~h}$ long treatments. Our current data indicate that if frequently repeated, even briefer FUS rounds would benefit precise and efficient treatment.

The effect of FUS-enhanced iTSL-DOX treatments was extended to the double-tumour model (Figure S8). As with the single tumour experiments, iTSL-DOX dosing causes a pronounced reduction in the tumour growth. This occurs even on the nil-FUS tumours but the most significant growth inhibition occurred when iTSL-DOX was combined with FUS. In this study mice also did not show critical weight loss even if they were bearing two tumours. The double tumour mouse model helps in assessing the effect of FUS in combination with the drug, as both tumours receive the dosing the same way and the effect of FUS can be seen more clearly.

Finally we investigated the effects on tumour apoptosis using double-tumour mice. The first group were injected with Annexin vivo 750 at $48 \mathrm{~h}$ after treatment with iTSL-DOX \pm FUS (omitting CF750.DSA to avoid fluorescence interference). Uptake of the apoptotic marker was assessed by NIRF imaging. The second received no marker but were sacrificed at the same time point with tumours excised, mounted, and $\mathrm{H} \& \mathrm{E}$ stained according to standard protocols [53]. Figure 10A shows one typical section of completely untreated tumour and one of FUS-only treated tumour. The stained nuclei indicate that the tumours were still viable but a necrotic tumour core is observed. Figure 10B demonstrates sections of tumours treated with iTSL-DOX with or without FUS. In all sections there were areas where nuclei were absent or unstructured (marked with arrows). These were observed at the periphery of the tumour with patches of dead cells forming evident areas of necrosis. Similarly, Annexin vivo 750 stained the FUS-treated tumour to a larger effect compared to the FUS-untreated tumour (Figure 10C), indicating that combination of iTSL-DOX and FUS enhanced tumour cell apoptosis. From the above in vivo studies it appears that iTSL-DOX released the drug rapidly and this high local dose was efficient in killing tumour cells.

Theranostic liposomes have repeatedly appeared in the literature for MR, PET, and SPECT imaging, as platforms for $\mathrm{pH}$ - and temperature-response triggered drug delivery, and for photodynamic or photothermal therapies [54]. These functional liposomes are likely the best developed nanomedicine but as functionalities increase there is a need for more sophisticated design. Anti-cancer nanomedicine has shown significant advances in the clinic over the last years with liposomal drugs reaching Phase-III trials [55][56]. As yet no MR-labelled or theranostic liposome has reached clinic but a MR-labelled polysiloxane gadolinium macrocycle has recently progressed to Phase-Ib for imaging and radiosensitisation - indicating the potential of these theranostic nanomedicines [57].
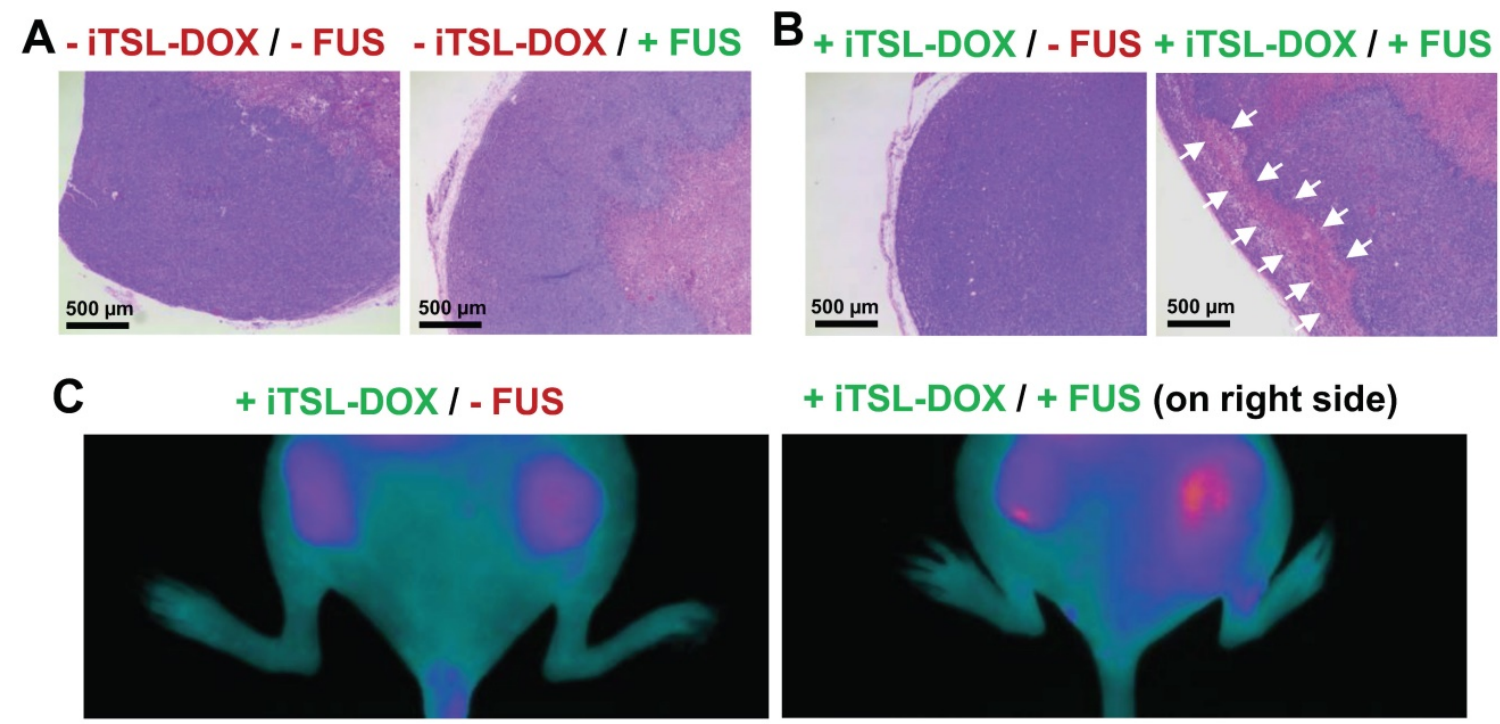

+ iTSL-DOX I + FUS (on right side)

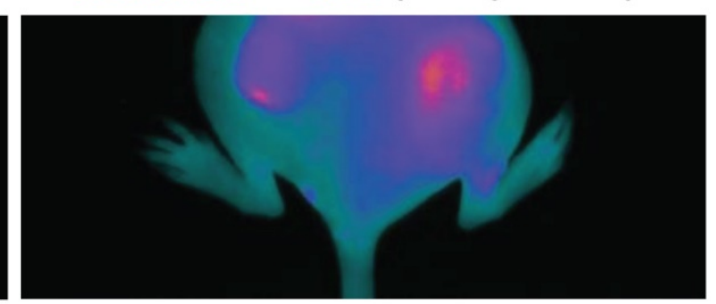

Figure 10. Assessing necrosis and apoptosis $48 \mathrm{~h}$ after treatment. (A) Histology $(\mathrm{H} \& \mathrm{E}$ sections), tumours were excised $48 \mathrm{~h}$ after the treatment. Slices $(4 \mu \mathrm{m})$ of MDA-MB-231 tumours untreated and (B) treated with iTSL-DOX and focused ultrasound (FUS) were stained with haematoxylin and eosin. The necrotic areas induced by the treatment are indicated by the arrows. Large necrotic areas could only be found when the combination of iTSL and hyperthermia was applied; (C) Apoptosis monitoring in living mice two days post treatment using Annexin vivo 750, with images recorded 3-4 h after the administration. Significant increases in apoptosis were apparent on the right side where FUS had been applied. 
Thermosensitive liposomal doxorubicin in the form of ThermoDox ${ }^{\circledR}$ is the most advanced responsive nanomedicine in clinical development. ThermoDox ${ }^{\circledR}$ was first-in-class but has not been as successful in clinic as was expected. Recent clinical trials have combined it with RFA (radiofrequency ablation) for liver tumours but in July 2020, Celsion announced that an independent Data Monitoring Committee recommended halting the Phase-III OPTIMA (NCT02112656) trial although the patients will be followed for overall survival. There may be several reasons for this apparent miss of OPTIMA's primary end point. One potential explanation is that since RFA ablates tumours, it may not be the best hyperthermia induction method to demonstrate clinical efficacy. FUS can offer controlled hyperthermia and when combined with MRI, the targeted tissue temperature is tracked during the treatment. ThermoDox ${ }^{\circledR}$ is planned to be combined in a clinical trial with FUS. This combination of ThermoDox® with a non-invasive hyperthermia technique may restore the clinical efficiency of TSL that is currently under question.

In this study we introduced MR-labelling onto ultrasound responsive liposomes to better control the timing of the thermally-triggered drug release. For this we synthesised a novel MR-lipid and took special attention to use a macrocyclic $\mathrm{Gd}^{3+}$ chelator that is considered to be substantially safe and it is widely used in clinical imaging [58]. We assessed the gadolinium retention under challenging conditions, and we evidence for the first time that these iTSL do not suffer significant $\mathrm{Gd}^{3+}$ leakage under in vivo relevant conditions and time frames. MRI studies with tumour-bearing mice demonstrated increased tumour $\mathrm{T}_{1}$ contrast for several hours post-injection of iTSL, suggesting that MR-monitoring of iTSL-DOX uptake into tumours will be feasible in the clinic. We also confirmed iTSL-DOX storage stability; a parameter critical for experimental product validation and for clinical translation.

Doxorubicin was selected as the therapeutic cargo. It is a hydrophilic drug, with a good ability to permeate membranes and distribute between tissue compartments [59]. It is rapidly cleared from tissues and blood when it is not encapsulated in liposomes. Although several studies have demonstrated the effect of hyperthermia-enhanced doxorubicin uptake by tumours, there is little or no information on clearance from FUS-treated versus non-treated tumours. In our experiments, NIRF imaging suggests that released doxorubicin does not remain in tumour tissue for long, so repetition of FUS rounds might benefit local dosing. Equivalent results were observed in our reported study for iTSL-topotecan [21]. Overall it seems iTSL+FUS has the potential to deliver drugs efficiently and specifically, and it is likely that a more potent anticancer agent would better suit this triggered release technology.

\section{Conclusions}

In summary, we have prepared novel MR-imageable liposomes with triggerable release properties, for precise FUS-mediated spatiotemporal tumour drug delivery with direct clinical relevance. The suggested iTSL-DOX is able to enhance MR $\mathrm{T}_{1}$ contrast of tumours in mice and provide a good signal for liposome tracking, which in turn allows for timely FUS application. The study shows that real-time imaging and brief FUS insonation combine to assist anticancer treatment to be precisely and correctly released such that a strong anti-tumour effect is achieved. This strategy has the potential advantages of (i) lowering the systemically administered chemotherapeutic dose, as the tumour fraction is dramatically increased; and (ii) providing tumour lesion contrast enhancement while being treated in real time. iTSL is a novel and versatile theranostic platform that could substantially improve chemotherapies' therapeutic index.

\section{Abbreviations}

CA: magnetic resonance imaging contrast agent; CF750: a near-infrared fluorescence dye; CF750.DSA: $N$-CF750- $N, N$-distearylamidomethylamine; DOTA: 1,4,7,10-tetraazacyclododecane-1,4,7,10-tetraacetic acid; DPPC: 1,2-dipalmitoyl-sn-glycero-3-phosphocholine; DSC: differential scanning calorimetry; DSL: dynamic light scattering; DSPC: 1,2-distearoyl-snglycero-3-phosphocholine;

DSPE-PEG ${ }^{2000}$ : ( $\omega$-methoxy-polyethyleneglycol ${ }^{2000}$ )- $N$-carboxy-1,2distearoyl-sn-glycero-3-phosphoethanolamine; DTPA: 2-[Bis[2-[bis(carboxymethyl)amino]ethyl]amino]acetic acid; ESI: electrospray ionisation; FBS: fetal bovine serum; FDA: food and drug administration; FOV: field of view; FUS: focused ultrasound; Gd.DOTA. DSA: gadolinium (III) 2-(4,7-bis-carboxymethyl-10[ $N, \quad N$-distearylamidomethyl-N'-amidomethyl]1,4,7,10-tetraazacyclododec-1-yl) acetic acid; HEPES: 2-[4-(2-hydroxyethyl)piperazin-1-yl]ethanesulfonic acid; HIFU: high intensity focused ultrasound; iTSL: imageable thermosensitive liposome; iTSL-DOX/NODOX: imageable thermosensitive liposome with/ without doxorubicin; LC-MS/MS: liquid chromatography with tandem mass spectroscopy; LED: light emitting diode; MR: magnetic resonance; MSPC: 1-stearoyl-sn-glycero-3-phosphocholine; MWCO: molecular weight cut-off; NIRF: near-infrared fluorescence; MRgFUS: magnetic resonance imaging guided focused ultrasound; PDI: polydispersity 
index; RO: reverse osmosis; ROI: region of interest; RT: room temperature; SHO: severe combined immunodeficiency hairless outbred; TNBC: triple-negative breast cancer; TSL: thermosensitive liposomes; TXRF: total-reflection X-ray fluorescence.

\section{Supplementary Material}

Supplementary figures and tables. http://www.ntno.org/v05p0125s1.pdf

\section{Acknowledgements}

This work was supported by the EPSRC (Engineering and Physical Sciences Research Council; grant EP/I001700/1), King's Commercialisation Institute, The Ministry of Education of Azerbaijan Republic, The London Metallomics Facility funded by the Wellcome Trust (grant 202902/Z/16/Z), EUNCL (European Nanotechnology Characterisation Lab) and Healthtech TAB (Translational Advisory Board). Special thanks also go to the Department of Chemistry, Biological Services Unit, Jayne Morgan, the Mass Spectroscopy Facility, and Anna Caldwell all at King's College London.

\section{Competing Interests}

The authors have declared that no competing interest exists.

\section{References}

1. Hahn GM. Potential for therapy of drugs and hyperthermia. Cancer Res. 1979; 39: 2264-68.

2. Zhu L, Altman MB, Laszlo A, Straube W, Zoberi I, Hallahan DE, et al. Ultrasound Hyperthermia Technology for Radiosensitization. Ultrasound Med Biol. 2019; 45: 1025-43.

3. Kennedy JE. High-intensity focused ultrasound in the treatment of solid tumours. Nat Rev Cancer. 2005; 5: 321-27.

4. Elhelf IAS, Albahar H, Shah U, Oto A, Cressman E, Almekkawy M. High intensity focused ultrasound: The fundamentals, clinical applications and research trends. Diagn Interv Imaging. 2018; 99: 349-59.

5. Fisher DG, Price RJ. Recent Advances in the Use of Focused Ultrasound for Magnetic Resonance Image-Guided Therapeutic Nanoparticle Delivery to the Central Nervous System. Front Pharmacol. 2019· 10: 1348

6. Basha SA, Salkho N, Dalibalta S, Husseini GA. Liposomes in Active, Passive and Acoustically-Triggered Drug Delivery. Mini Rev Med Chem. 2019; 19: 961-69.

7. Karimi M, Sahandi Zangabad P, Ghasemi A, Amiri M, Bahrami M, Malekzad $\mathrm{H}$, et al. Temperature-Responsive Smart Nanocarriers for Delivery Of Therapeutic Agents: Applications and Recent Advances. ACS Appl Mater Interfaces. 2016; 8: 21107-33.

8. Needham D, Dewhirst MW. The development and testing of a new temperature-sensitive drug delivery system for the treatment of solid tumors. Adv Drug Deliv Rev. 2001; 53: 285-305.

9. Needham D, Anyarambhatla G, Kong G, Dewhirst MW. A new temperaturesensitive liposome for use with mild hyperthermia: characterization and testing in a human tumor xenograft model. Cancer Res. 2000; 60: 1197-1201.

10. Haemmerich D, Motamarry A. Thermosensitive Liposomes for Image-Guided Drug Delivery. Adv Cancer Res. 2018; 139: 121-46.

11. Dewhirst MW, Landon CD, Hofmann CL, Stauffer PR. Novel approaches to treatment of hepatocellular carcinoma and hepatic metastases using thermal ablation and thermosensitive liposomes. Surg Oncol Clin N Am. 2013; 22: 545-61.

12. Kono K, Nakashima S, Kokuryo D, Aoki I, Shimomoto H, Aoshima S, et al. Multi-functional liposomes having temperature-triggered release and magnetic resonance imaging for tumor-specific chemotherapy. Biomaterials. 2011; 32: 1387-95.

13. Aoki I, Yoneyama M, Hirose J, Minemoto $\mathrm{Y}$, Koyama T, Kokuryo D, et al. Thermoactivatable polymer-grafted liposomes for low-invasive image-guided chemotherapy. Transl Res. 2015; 166: 660-673 e1.
14. Viglianti BL, Abraham SA, Michelich CR, Yarmolenko PS, MacFall JR, Bally $\mathrm{MB}$, et al. In vivo monitoring of tissue pharmacokinetics of liposome/drug using MRI: illustration of targeted delivery. Magn Reson Med. 2004; 51: 1153-62.

15. Tang WL, Tang WH, Li SD. Cancer theranostic applications of lipid-based nanoparticles. Drug Discov Today. 2018; 23: 1159-66.

16. Hijnen N, Kneepkens E, de Smet M, Langereis S, Heijman E, Grull H. Thermal combination therapies for local drug delivery by magnetic resonance-guided high-intensity focused ultrasound. Proc Natl Acad Sci U S A. 2017; 114: E4802-11.

17. Hijnen N, Langereis S, Grüll H. Magnetic resonance guided high-intensity focused ultrasound for image-guided temperature-induced drug delivery. Adv Drug Del Rev. 2014; 72: 65-81.

18. Rizzitelli S, Giustetto P, Faletto D, Delli Castelli D, Aime S, Terreno E. The release of Doxorubicin from liposomes monitored by MRI and triggered by a combination of US stimuli led to a complete tumor regression in a breast cancer mouse model. J Control Release. 2016; 230: 57-63.

19. Celik H, Wakim P, Pritchard WF, Castro M, Leonard S, Karanian JW, et al Radiofrequency Ablation Duration per Tumor Volume May Correlate with Overall Survival in Solitary Hepatocellular Carcinoma Patients Treated with Radiofrequency Ablation Plus Lyso-Thermosensitive Liposomal Doxorubicin. J Vasc Interv Radiol. 2019; 30: 1908-14

20. Lyon PC, Gray MD, Mannaris C, Folkes LK, Stratford M, Campo L, et al. Safety and feasibility of ultrasound-triggered targeted drug delivery of doxorubicin from thermosensitive liposomes in liver tumours (TARDOX): a single-centre, open-label, phase 1 trial. Lancet Oncol. 2018; 19: 1027-39.

21. Centelles MN, Wright M, So PW, Amrahli M, Xu XY, Stebbing J, et al. Imageguided thermosensitive liposomes for focused ultrasound drug delivery: Using NIRF-labelled lipids and topotecan to visualise the effects of hyperthermia in tumours. J Control Release. 2018; 280: 87-98.

22. Rosca E V., Wright M, Gonitel R, Gedroyc W, Miller AD, Thanou M. Thermosensitive, near-infrared-labeled nanoparticles for topotecan delivery to tumors. Mol Pharm. 2015; 12: 1335-46.

23. Ashraf A, Stosnach H, Parkes HG, Hye A, Powell J, So PW, et al. Pattern of Altered Plasma Elemental Phosphorus, Calcium, Zinc, and Iron in Alzheimer's Disease. Sci Rep. 2019; 9: 3147

24. Rohrer M, Bauer H, Mintorovitch J, Requardt M, Weinmann HJ. Comparison of magnetic properties of MRI contrast media solutions at different magnetic field strengths. Invest Radiol. 2005; 40: 715-24.

25. Kamaly N, Kalber T, Ahmad A, Oliver MH, So PW, Herlihy AH, et al. Bimodal paramagnetic and fluorescent liposomes for cellular and tumor magnetic resonance imaging. Bioconjug Chem. 2008; 19: 118-29.

26. Marasini R, Thanh Nguyen TD, Aryal S. Integration of gadolinium in nanostructure for contrast enhanced-magnetic resonance imaging. Wiley Interdiscip Rev Nanomed Nanobiotechnol. 2020; 12: e1580.

27. Sadeghi N, Deckers R, Ozbakir B, Akthar S, Kok RJ, Lammers T, et al. Influence of cholesterol inclusion on the doxorubicin release characteristics of lysolipid-based thermosensitive liposomes. Int J Pharm. 2018; 548: 778-82.

28. Demetzos C. Differential Scanning Calorimetry (DSC): A Tool to Study the Thermal Behavior of Lipid Bilayers and Liposomal Stability. J Liposome Res. 2008; 18: 159-73.

29. Sandstrom MC, Ickenstein LM, Mayer LD, Edwards K. Effects of lipid segregation and lysolipid dissociation on drug release from thermosensitive liposomes. J Control Release. 2005; 107: 131-42.

30. Sandström MC, Ickenstein LM, Mayer LD, Edwards K. Effects of lipid segregation and lysolipid dissociation on drug release from thermosensitive liposomes. J Control Release. 2005; 107: 131-42.

31. Park SM, Kim MS, Park SJ, Park ES, Choi KS, Kim YS, et al. Novel temperature-triggered liposome with high stability: Formulation, in vitro evaluation, and in vivo study combined with high-intensity focused ultrasound (HIFU). J Control Release. 2013; 170: 373-79.

32. Kneidl B, Peller M, Winter G, Lindner LH, Hossann M. Thermosensitive liposomal drug delivery systems: state of the art review. Int J Nanomedicine. 2014; 9: 4387-98.

33. Banno B, Ickenstein LM, Chiu GN, Bally MB, Thewalt J, Brief E, et al The functional roles of poly(ethylene glycol)-lipid and lysolipid in the drug retention and release from lysolipid-containing thermosensitive liposomes in vitro and in vivo. J Pharm Sci. 2010; 99: 2295-2308.

34. Guntur SR, Choi MJ. An improved tissue-mimicking polyacrylamide hydrogel phantom for visualizing thermal lesions with high-intensity focused ultrasound. Ultrasound Med Biol. 2014; 40: 2680-91.

35. Rogosnitzky M, Branch S. Gadolinium-based contrast agent toxicity: a review of known and proposed mechanisms. Biometals. 2016; 29: 365-76.

36. Szomolanyi P, Rohrer M, Frenzel T, Noebauer-Huhmann IM, Jost G, Endrikat $\mathrm{J}$, et al. Comparison of the Relaxivities of Macrocyclic Gadolinium-Based Contrast Agents in Human Plasma at 1.5, 3, and 7 T, and Blood at $3 \mathrm{~T}$. Invest Radiol. 2019; 54: 559-64.

37. Rohrer M, Bauer H, Mintorovitch J, Requardt M, Weinmann H-J. Comparison of Magnetic Properties of MRI Contrast Media Solutions at Different Magnetic Field Strengths. Biomaterials. 2005; 40: 715-24.

38. Rogosnitzky M, Branch S. Gadolinium-based contrast agent toxicity: a review of known and proposed mechanisms. Biometals. 2016; 29: 365-76.

39. Huynh K, Baghdanian AH, Baghdanian AA, Sun DS, Kolli KP, Zagoria RJ. Updated guidelines for intravenous contrast use for CT and MRI. Emerg Radiol. 2020; 27: 115-26. 
40. Clough TJ, Jiang L, Wong KL, Long NJ. Ligand design strategies to increase stability of gadolinium-based magnetic resonance imaging contrast agents. Nat Commun. 2019; 10: 1420 .

41. Frenzel T, Lengsfeld P, Schirmer H, Hutter J, Weinmann HJ. Stability of gadolinium-based magnetic resonance imaging contrast agents in human serum at 37 degrees C. Invest Radiol. 2008; 43: 817-28.

42. Prybylski JP, Semelka RC, Jay M. The stability of gadolinium-based contrast agents in human serum: A reanalysis of literature data and association with clinical outcomes. Magn Reson Imaging. 2017; 38: 145-51.

43. Gabizon A, Shmeeda H, Barenholz Y. Pharmacokinetics of pegylated liposomal Doxorubicin: review of animal and human studies. Clin Pharmacokinet. 2003; 42: 419-36.

44. Liu C, Ewert KK, Wang N, Li Y, Safinya CR, Qiao W. A multifunctional lipid that forms contrast-agent liposomes with dual-control release capabilities for precise MRI-guided drug delivery. Nucl Med Mol Imaging. 2019; 221: 119412.

45. Santos MA, Goertz DE, Hynynen K. Focused ultrasound hyperthermia mediated drug delivery using thermosensitive liposomes and visualized with in vivo two-photon microscopy. Theranostics. 2017; 7: 2718-31.

46. Motamarry A, Negussie AH, Rossmann C, Small J, Wolfe AM, Wood BJ, et al. Real-time fluorescence imaging for visualization and drug uptake prediction during drug delivery by thermosensitive liposomes. Int J Hyperth. 2019; 36: 817-26.

47. de Smet M, Langereis S, van den Bosch S, Bitter K, Hijnen NM, Heijman E, et al. SPECT/CT imaging of temperature-sensitive liposomes for MR-image guided drug delivery with high intensity focused ultrasound. J Control Release. 2013; 169: 82-90.

48. Tak WY, Lin SM, Wang Y, Zheng J, Vecchione A, Park SY, et al. Phase III HEAT study adding lyso-thermosensitive liposomal doxorubicin to radiofrequency ablation in patients with unresectable hepatocellular carcinoma lesions. Clin Cancer Res. 2018; 24: 73-83.

49. Sanches PG, Grüll H, Steinbach OC. See, reach, treat: Ultrasound-triggered image-guided drug delivery. Ther Deliv. 2011; 2: 919-34.

50. Kuroda K. MR techniques for guiding high-intensity focused ultrasound (HIFU) treatments. J Magn Reson Imaging. 2018; 47: 316-31.

51. Yi XM, Wang FL, Qin WJ, Yang XJ, Yuan JL. Near-infrared fluorescent probes in cancer imaging and therapy: An emerging field. Int J Nanomedicine. 2014; 9: $1347-65$.

52. Lokerse WJM, Bolkestein M, Dalm SU, Eggermont AMM, de Jong M, Grüll H, et al. Comparing the therapeutic potential of thermosensitive liposomes and hyperthermia in two distinct subtypes of breast cancer. J Control Release. 2017; 258: $34-42$.

53. Fischer AH, Jacobson KA, Rose J, Zeller R. Hematoxylin and eosin staining of tissue and cell sections. Cold Spring Harb Protoc. 2008; 3.

54. Lee W, Im HJ. Theranostics Based on Liposome: Looking Back and Forward. Clin Transl Med. 2019; 53: 242-46.

55. Germain M, Caputo F, Metcalfe S, Tosi G, Spring K, Åslund AKO, et al. Delivering the power of nanomedicine to patients today. J Control Release. 2020; 326: 164-71.

56. Tran S, DeGiovanni P-J, Piel B, Rai P. Cancer nanomedicine: a review of recent success in drug delivery. Clin Toxicol. 2017; 6: 44

57. Lux F, Tran VL, Thomas E, Dufort S, Rossetti F, Martini M, et al. AGuiX ® from bench to bedside-transfer of an ultrasmall theranostic gadolinium-based nanoparticle to clinical medicine. Br J Radiol. 2019; 92.

58. Layne KA, Wood DM, Dargan PI. Gadolinium-based contrast agents - what is the evidence for 'gadolinium deposition disease' and the use of chelation therapy? Clin Toxicol. 2020; 58: 151-60.

59. Zhan W, Gedroyc W, Xu XY. The effect of tumour size on drug transport and uptake in 3-D tumour models reconstructed from magnetic resonance images. PLoS One. 2017; 12: e0172276. 